\title{
A Wnt-specific astacin proteinase controls head formation in Hydra
}

Berenice Ziegler ${ }^{1}$, Irene Yiallouros $^{2}$, Benjamin Trageser $^{1}$, Sumit Kumar ${ }^{3}$, Moritz Mercker $^{4}$, Svenja Kling ${ }^{1}$, Maike Fath ${ }^{1}$, Uwe Warnken ${ }^{5}$, Martina Schnölzer ${ }^{5}$, Thomas W. Holstein'1, Markus Hart|' ${ }^{6}$ Anna Marciniak-Czochra ${ }^{4}$, Jörg Stetefeld", Walter Stöcker² and Suat Özbek ${ }^{1 *}$

${ }^{1}$ University of Heidelberg, Centre for Organismal Studies, Department of Molecular Evolution and Genomics, Im Neuenheimer Feld 230, 69120 Heidelberg, Germany. 2Institute of Molecular Physiology, Cell and Matrix Biology, Johannes Gutenberg University Mainz, 55099, Mainz, Germany.

${ }^{3}$ Department of Cell and Chemical Biology, Leiden University Medical Center, Netherlands

${ }^{4}$ Institute for Applied Mathematics, Interdisciplinary Center for Scientific Computing, Heidelberg University, Im Neuenheimer Feld 205, 69120, Heidelberg, Germany.

${ }^{5}$ Functional Proteome Analysis, German Cancer Research Center (DKFZ), Heidelberg, Germany

${ }^{6}$ Institute of Biochemistry and Center for Molecular Biosciences, University of Innsbruck, Innrain 80-82, A-6020 Innsbruck, Austria.

${ }^{7}$ Department of Chemistry, University of Manitoba, 144 Dysart Road, Winnipeg, Manitoba, Canada R3T 2N2

*Address correspondence to: Suat Özbek, University of Heidelberg, Centre for Organismal Studies, Department of Molecular Evolution and Genomics, Im Neuenheimer Feld 230, 69120 Heidelberg, Germany. 


\section{Abstract}

The Hydra head organizer acts as a signaling center that initiates and maintains the primary body axis in steady state polyps and during budding or regeneration. Wnt/betaCatenin signaling functions as a primary cue controlling this process, but how Wnt ligand activity is locally restricted at the protein level is poorly understood.

Here we report the identification of an astacin family proteinase as a Wnt processing factor. Hydra astacin-7 (HAS-7) is expressed from gland cells as an apical-distal gradient in the body column, peaking close beneath the tentacle zone. HAS-7 siRNA knockdown abrogates HyWnt3 proteolysis in the head tissue and induces a robust double axis phenotype, which is rescued by simultaneous HyWnt3 knockdown. Accordingly, double axes are also observed in conditions of increased Wnt levels as in transgenic actin::HyWnt3 and HyDkk1/2/4 siRNA treated animals. HyWnt3-induced double axes in Xenopus embryos could be rescued by co-injection of HAS-7 mRNA. Mathematical modelling combined with experimental promotor analysis indicate an indirect regulation of HAS-7 by beta-Catenin, expanding the classical Turing-type activator-inhibitor model.

Our data suggest a negative regulatory function of Wnt processing astacin proteinases in the global patterning of the oral-aboral axis in Hydra. 


\section{Introduction}

Wnt ligands secreted from a local source at the posterior end of the embryo are conserved molecular cues for the patterning of the primary body axis in bilaterians and non-bilaterians [1-3]. The role of Wnt/beta-Catenin signaling in the axial patterning of cnidarians has been extensively studied in the freshwater polyp Hydra [4-7], which has a single oral-aboral body axis. The head is separated from the gastric region by a ring of tentacles and runs out at the upper part into a cone-shaped mouth region, called the hypostome. At its apical tip the hypostome contains the head organizer [8] comprising a small cluster of ecto- and endodermal cells that continuously express HyWnt3 in steady state polyps (Fig. 1a) [9]. HyWnt3 is upregulated early during head regeneration and has been shown to initiate a cascade of Wnt signaling events directing axial patterning [7]. While the spatially restricted HyWnt3 ligand production is controlled at the transcriptional level by repressive elements in the HyWnt3 promotor region [9, 10], it is poorly understood how Wnt activity is regulated at protein level in the extracellular space. In Hydra, only a member of the Dkk1/2/4 family of secreted Wnt inhibitors has so far been shown to function as a Wnt antagonist by creating a Wnt-suppressed region in the body column [11]. Recently, we have shown that the matricellular protein Thrombospondin (HmTSP) is expressed directly from or in close vicinity of HyWnt3 expressing cells of the hypostome and exerts a negative regulatory function on organizer formation [12]. It is unclear, though, whether HmTSP interacts directly with Wnt ligands or modulates Wnt inactivity by influencing receptor mobility or turnover. Morphogen activity during embryogenesis can also be restricted by proteinases that process secreted ligands. A prominent example is the zinc metalloproteinase BMP1 and its splice variant mammalian Tolloid (i.e. Xolloid in Xenopus), which specifically cleaves Chordin and thus promotes local BMP signaling at the ventral side of the 
vertebrate embryo [13]. A similar case for morphogen inactivation has been proposed for TIKI1, a highly conserved metalloproteinase expressed in the Xenopus organizer and shown to antagonize Wnt function by cleaving eight amino-terminal residues of Wnt3a [14].

In Hydra, functional studies on astacin metalloproteinases have indicated important roles in processes of morphogenesis and regeneration [15-17]. Yan et al. have shown that the metalloproteinase HMP1 is localized to the head pole and an anti-HMP1 antibody can effectively block head regeneration [17]. HMP2, a Hydra astacin proteinase of the meprin family, formed an opposing gradient to HMP1, showing highest expression at the basal pole of the animal [16]. Although different mechanistic pathways as the proteolytic activation of morphogens or regulatory peptides have been discussed in these studies, no detailed molecular mechanisms comparable to those for Tolloid or TIKI1 have been described so far for any cnidarian metalloproteinase. Here, we identify a member of the astacin proteinase family in Hydra with Wnt3 processing activity. Hydra Astacin-7 (HAS-7) is expressed in an increasing gradient towards the tentacle base of the polyp, forming a ring-like zone between head and body column that shows upregulated expression for several other members of the astacin family. siRNA knockdown of HAS-7 eliminates the HyWnt3 proteolytic activity of the head tissue leading to a robust double axis phenotype with a fully developed head structure. In addition, HAS-7 mRNA injection into Xenopus embryos rescues double axes induced by HyWnt3 mRNA. Our combined experimental data and mathematical models demonstrate a direct mechanistic link between astacin proteinases and Wnt-regulated pattern formation in Hydra by restricting Wnt ligand activity to the head region via specific proteolysis. 


\section{Results and Discussion}

\section{Identification of HyWnt3 proteolytic activity in Hydra head lysate}

To identify factors restricting Wnt activity in the extracellular space we first examined the protein half-life of recombinant HyWnt3-His in tissue lysates generated from different body parts of Hydra (Fig. 1a). For this, lysates of the head region, tentacles, upper and lower body trunk were prepared and their soluble fractions were adjusted in total protein concentration to $4 \mathrm{mg} / \mathrm{ml}$. $\sim 10 \mathrm{ng}$ of purified recombinant HyWnt3-His protein was incubated with equal amounts of each tissue lysate and the reaction was stopped after different time points. Detection by Western blotting located the highest proteolytic activity for HyWnt3-His in the head (half-life $\sim 4$ h) and, to a lesser extent, upper body lysates (half-life $\sim 6$ hrs) (Fig. 1b, d). While unspecific proteolysis of HyWnt3-His was evident after 24 hrs incubation in PBS, it stayed remarkably stable in lysates of tentacles and the lower body part. Incubation of $1 \mu \mathrm{g} \mathrm{BSA}$ in head lysate (HL) did not show unspecific proteolysis over the given time period of 24 hrs (Fig. 1b). In HL samples supplemented with broad spectrum metalloproteinase inhibitors like EDTA and 1,10-Phenanthroline or the specific matrix metalloproteinase inhibitor Batimastat, HyWnt3-His processing was completely blocked in the given time frame, indicating that metalloproteinases could be responsible for the observed activity. A parallel experiment performed with recombinantly expressed HyDkk1/2/4-His protein, a major Wnt antagonist [11], showed no specific proteolytic activity for this factor in the respective lysates (Fig. 1c, d).

To isolate candidate factors involved in HyWnt3 processing we next used a proteomic approach. A pool of $\mathrm{HL}$ from 200 polyps was fractionated by cation exchange chromatography (Additional file 1: Fig. S1a) and peak fractions were re-examined for their HyWnt3-His processing activity applying a $6 \mathrm{hrs}$ incubation time (Additional file 1: 
Fig. S1b). A fragment encompassing the two N-terminal cadherin domains of Hydra cadherin [18] was used as a control substrate to monitor general matrix metalloproteinase activity. We observed complete HyWnt3-His cleavage using fractions 1-5, while Hydra cadherin was degraded partially by fractions 2 and 3 . To exclude a high background of possibly unspecific proteinases in fractions 1-3 we pooled fractions 4-5 (HyWnt3(+)) and 6-7 (HyWnt3(-)) for further analysis and performed orbitrap mass spectrometry analysis after in solution digestion of the respective pooled samples. When we filtered the obtained protein hits for unique sequences of proteins having a signal peptide for secretion and at least two peptide hits, astacin family proteinases constituted the largest group in the HyWnt3(+) secretome while miscellaneous enzymes dominated in the HyWnt3(-) fraction (Fig. 1e, Additional file 4: Table S1a-b, Additional file 5: Table S2). Of the 12 astacin sequences detected in the HyWnt3(+) fraction, 4 were also present in the HyWnt3(-) fraction, although with lower protein scores. The HyWnt3(-) secretome additionally contained an increased number of proteinases belonging to diverse families (Additional file 4: Table S1b, Additional file 5: Table S2). We concluded from these results that metalloproteinases, in particular astacin-type proteinases, are likely candidates for the observed HyWnt3-His processing activity. To confirm this notion, we tested the proteolytic activity of $\mathrm{HL}$ on HyWnt3 in the presence of recombinant mammalian Fetuin-B, which was recently shown to function as a highly specific physiological inhibitor of astacin-type proteinases like ovastacin [19]. As shown in Fig. 1f, murine Fetuin-B blocked HyWnt3-His processing by $\mathrm{HL}$ in a dose-dependent manner. 


\section{Characterization of the HyWnt3(+) astacin secretome}

The HL HyWnt3(+) secretome contained 12 unique astacin sequences (hence called Hydra Astacins, HAS) with HAS-1 and HAS-7 showing the highest protein scores in the orbitrap mass spectrometry analysis (Additional file 4: Table S1a, Additional file 5: Table S2). The alignment of the pro- and catalytic domains with known astacin proteinase amino acid sequences demonstrated a high conservation of critical sequence motifs as the aspartate switch residue, methionine turn, and zinc binding motif (Fig. 2a). The domain structure of astacins comprises a signal peptide and a variable pro-domain segment, which is cleaved to activate the central 200 -residue catalytic domain (Fig. 2a-b). Typical for cnidarian astacins is the possession of Cterminal ShKT (Stichodactyla toxin) domains [22]. The majority of the astacins detected in our analysis comprises 1-2 ShKT domains, but several lack a C-terminal segment (Fig. 2b). HAS-11 is exceptional in possessing six ShKT domains in a tandem repeat. None of the astacin sequences was predicted to possess a transmembrane domain. To be able to correlate the observed HyWnt3-specific proteolytic activity in the head and upper body tissues with expression patterns of the respective genes, we performed in situ hybridization experiments in whole mounts (WISH). Conventional WISH using mRNA antisense probes and sense controls was performed for the genes encoding the two highest-scoring protein hits, HAS-1 and HAS-7, as well as for the previously described HMP1 [16]. For the remaining astacin genes of the Wnt3(+) fraction, synthetic locked nucleic acid (LNA) probes were used that have an increased target specificity for highly similar gene sequences. ISH probe and LNA sequences are summarized in Additional file 6: Table S3. The majority of astacin genes showed an endodermal expression pattern in the upper half of the body trunk, often as a gradient that increased towards the head with a sharp boundary beneath the tentacle formation 
zone (HAS-2, HAS-7, HMP1, Fig. $2 \mathrm{c}-\mathrm{d}$, f), reminiscent of the SEC-1 positive gland cell subpopulation described by Schmidt \& David [23]. Several astacins (HAS-5, HAS-911) were expressed in a narrow collar demarcating the body region from the head (Fig. $2 g-j)$. Four of the examined genes did not yield a reproducible staining pattern by WISH. HAS-1 was exceptional in exhibiting a distinct expression in large gland cells in the endoderm that were homogenously distributed along the entire body column (Fig. 2e). This pattern is reminiscent of the Wnt antagonist $H y D k k 1 / 2 / 4$, which is expressed by a subpopulation of zymogen gland cells [11]. By tracing the astacin genes of the HyWnt3(+) fraction in the Hydra single cell transcriptome database provided by the Celina Juliano lab (https://portals.broadinstitute.org/single_cell/study/stem-celldifferentiation-trajectories-in-hydra-resolved-at-single-cell-resolution) [24] we were able to identify cell-specific expression signatures for each sequence (Additional file 2: Fig. S2a-b). Scatter plots showing the expression profiles along cell types and differentiation trajectories clearly identify i-cell-derived gland cells distributed mostly along the transition zone between head and upper body (zymogen to mucous gland cell transition) as the source of all examined astacins, except $H A S-1$, which was restricted to zymogen gland cells of the body column. A close-up view of the epithelial layers in the upper gastric region of a HAS-7 WISH sample confirmed that the mRNA signal is not present in the endodermal epithelial cells closely aligning the central line of extracellular matrix, called mesoglea (Additional file 2: Fig. S2c). Gland cells in Hydra are interspersed singly between the endodermal epithelial cells without direct contact to the mesoglea [23]. In summary, the candidate astacin proteinases identified in our analysis are a product of endodermal i-cell derived gland cells with an overlapping peak expression beneath the head and tentacle region. We focused our further analysis on HAS-7 due to its high protein score in the mass spectrometry 
analysis and the graded expression pattern reflecting the diminishing HyWnt3-His proteolysis from head to lower body (Fig. 1a). Also, six of the astacins identified in the HyWnt3(+) HL fraction were also contained in the HyWnt3(-) fraction (HAS-1-3, HAS6, HAS-9, HAS-11), indicating that they are unlikely candidates for acting as HyWnt3specific proteinases. Interestingly, none of the astacins identified in both fractions belongs to the group showing a graded apical-to-basal expression pattern.

\section{HAS-7 knockdown induces double axis formation}

We next asked whether HAS-7 depletion perturbs normal axis formation as a consequence of diminished HyWnt3 processing. siRNA gene expression knockdown by electroporation of adult polyps has recently been established as a robust method in Hydra $[12,25,26]$. We designed siRNAs targeting conserved motifs in the HAS-7 prodomain (siRNA1), catalytic domain (siRNA2) and a less conserved region in the Cterminal ShKT domain (siRNA3) (sequences are given in Additional file 7: Table S4). To be able to monitor the electroporation efficiency in the endoderm we used an endodermal actin::GFP/ecdodermal actin::RFP transgenic strain (reverse watermelon) and applied siGFP in combination with target gene siRNAs [12, 25]. Although the knockdown phenotype for the strongly expressed GFP transgene is largely half-sided, we have evidence from previous studies that for native target genes the effect can be systemic [26]. To monitor the gene knockdown at protein level, we raised a polyclonal antibody against a unique epitope in the HAS-7 primary sequence located between the catalytic domain and the N-terminal ShKT domain (APPTAGPTISPT). A Western blot analysis of the different body part lysates used for the initial proteolytic assay showed a broad band migrating at $\sim 40 \mathrm{kDa}$, which was strongest in the head tissue lysate (Fig. 3a). Upper and lower body tissues showed diminished signals reflecting 
the decreasing HyWnt3-His proteolytic activity observed in the respective body parts (Fig. 1b). The calculated molecular mass of full-length HAS-7 is $37.9 \mathrm{kDa}$, the mature proteinase lacking signal and pro-peptide sequences is predicted to have $33.3 \mathrm{kDa}$. Upper and lower body lysates showed additional bands at 55 and $70 \mathrm{kDa}$, which might be the result of a cross-reaction with a related epitope sequence, or represent a dimeric form of mature HAS-7 as described previously for meprins [27]. A recombinant histidine-tagged version of HAS-7 protein expressed in the insect cell line High Five could be detected by the specific Has-7 antibody (Fig. 3b, Additional file 3: Fig. S3). The apparent molecular mass of recombinant HAS-7 was slightly higher compared to the main band in the $\mathrm{HL}$ reflecting the additional amino acids of the tag sequence (1.7 kDa). siRNA knockdown with a combination of siRNAs $1 / 2$ led to a moderate reduction of the $40 \mathrm{kDa}$ band intensity as detected in whole body lysates (Fig. 3c). The combination of siRNAs $2 / 3$ completely reduced band signals in this region and at the $70 \mathrm{kDa}$ position. The $55 \mathrm{kDa}$ band showed equal intensity in all conditions suggesting that it likely represents an unspecific protein. When we monitored HyWnt3-His half-life in head lysates of HAS-7 siRNAs 2/3 versus siGFP-treated animals, HyWnt3-His processing in head lysates after HAS-7 knockdown was significantly impaired (Fig. 3de), suggesting that HAS-7 proteinase is responsible for the observed HyWnt3 cleavage in Hydra. The siRNA knockdown was additionally confirmed at the transcriptional level by quantitative RT-PCR analysis of head tissue from siGFP and siHAS-7 treated animals (Fig. 3f).

In steady-state hydras, HAS-7 mRNA depletion led to the induction of ectopic axis with a fully developed head structure in about $50 \%$ of the animals (Fig. 3i, o). To confirm that this is due to an increased Wnt signaling level we challenged electroporated hydras by treatment with $A Z K$, a glycogen synthase kinase-3 $\beta$ inhibitor that leads to a 
systemic increase of beta-Catenin activity. This treatment normally induces ectopic tentacles along the gastric region 2-3 days after AZK removal emanating from small spot-like organizers that transiently express HyWnt3 (Fig. 3h) [4]. siHAS-7 electroporated animals that additionally received an AZK pulse showed fewer ectopic tentacles, but formed double axes at a constant rate of $80-90 \%$ as compared to siGFP electroporated polyps, indicating that this phenotype dominates under conditions of increased basal levels of beta-Catenin activity (Fig. $3 \mathrm{~h}-\mathrm{i}, \mathrm{o})$. A transgenic reporter line expressing eGFP under control of the HyWnt3 promotor (HyWnt3P::eGFP) [9] confirmed that the ectopic head induced by HAS-7 siRNA knockdown harbors a functional head organizer with hypostomal HyWnt3 expression (Fig. 3j). Interestingly, a double and often multiple axis morphology was also observed in a transgenic strain that expresses HyWnt3 under control of the actin promoter in all endodermal epithelial cells (Fig. 3k). Double axes were also obtained when animals were electroporated with siRNAs targeting the Wnt antagonist HyDkk1/2/4 (Fig. 3l), although animals in this group regularly showed in addition multiple ectopic tentacles along the body column. In summary, depletion by siRNA knockdown efficiently reduced HAS-7 protein levels as well as HyWnt3-His proteolytic activity of the respective $\mathrm{HL}$ and resulted in an almost exclusive double axis phenotype that in AZK-treated animals. This is likely a consequence of unphysiologically increased HyWnt3 activity as similar phenotypes were observed by transgenic HyWnt3 overexpression or HyDkk1/2/4 depletion. This assumption was tested by combining HyWnt3 and HAS-7 siRNAs under AZK conditions, which resulted in a complete reversal of the double axis phenotype, although ectopic tentacles were still formed due to the increased beta-Catenin activity (Fig. 3m, o). Interestingly, HMP-1 siRNA knockdown combined with AZK treatment also resulted in ectopic axis induction, but at a substantially lower level than for HAS- 
7 (Fig. 30), indicating some redundancy among astacins with similar expression patterns. No secondary axes were observed in animals electroporated with HyDkk and HMP-1 siRNAs without subsequent AZK treatment. siRNA knockdown of HAS-1, which does not exhibit a graded apical-distal expression pattern, did not result in double axis formation after AZK treatment $(n, 0)$. To further confirm that HAS-7 is responsible for HyWnt3 inactivation in vivo we made use of the standard axial duplication assay in Xenopus laevis. Injection of $10 \mathrm{pg}$ XWnt8 mRNA in each blastomere of four-cell embryos induced secondary axis formation in more than $60 \%$ of the embryos compared to controls (Fig. 3p). Although HyWnt3 injection induced secondary axes as well, higher mRNA doses (100 pg) were necessary to reach a comparable level to XWnt8. Co-injection of $100 \mathrm{pg}$ HAS-7 mRNA inhibited HyWnt3-induced secondary axis formation, indicating that HAS-7 is sufficient to antagonize HyWnt3 function in a heterologous setting.

\section{beta-Catenin dependency of HAS-7 expression}

The HAS-7 expression zone, similar to the one of HyDkk1/2/4 [11], is distinctly excluded from the region of Wnt expression in the hypostome area. This questions a direct beta-Catenin/TCF-dependent regulation of HAS-7 as previously demonstrated for HmTSP that is expressed by HyWnt3-positive cells of the organizer [12]. When HAS-7 gene expression was monitored by WISH following Alsterpaullone (ALP) treatment, we observed a global increase within the gastric region after $24 \mathrm{hrs,}$ resulting in a loss of the original apical-distal expression gradient (Fig. 4a-b). 48 hrs after ALP wash, a line of increased HAS-7 expression was evident close to regions of ectopic tentacle formation (Fig. 4c). In contrast to global activation by ALP, betacatenin inhibition by iCRT14 treatment [28] did not alter the HAS-7 expression pattern 
(Fig. 4e), arguing against a direct regulation by beta-Catenin/TCF. In transgenic actin::HyWnt3 Hydra, HAS-7 was strongly upregulated throughout the gastric region (Fig. 4f), confirming that the observed increase of HAS-7 expression induced by ALP is likely a consequence of global beta-Catenin activation. Interestingly, the head region was free of HAS-7 mRNA in actin::HyWnt3 transgenic animals.

Upon examination of the HAS-7 promoter region for regulatory elements, we identified a single putative TCF binding element with the conserved sequence motif 5'CTTTGTT3' (Fig. 4g), similar to those experimentally confirmed in the HyWnt3 [9] and HmTSP [12] promoters (CTTTGWW, W=A or T). To gain evidence whether HAS7 is directly regulated by beta-Catenin/TCF, we performed a chromatin immunoprecipitation (ChIP) assay using a Hydra TCF-specific antiserum as described previously $[12,29]$. Chromatin was taken from control polyps and ALP-treated animals. No PCR product was obtained with primers flanking the identified TCF binding element in the HAS-7 promoter in control and ALP conditions (Fig. 4g). In contrast, amplification of the TCF binding element in the HmTSP promoter yielded the expected PCR product from both samples (Fig. 4h). This suggests that TCF is not bound to the putative DNA binding region in the HAS-7 promoter both in steady-state polyps and under conditions of global increase in nuclear beta-Catenin. We therefore presume that $H A S-7$ is an indirect downstream target of beta-Catenin/TCF, which is positively regulated as part of the global Wnt signaling response, but is controlled by additional factors that downregulate its expression in the vicinity of Wnt producing cells.

\section{HAS-7 function ensures single organizer dominance in an extended Turing model}

To investigate the role of HAS-7 during axis and head pattern formation in Hydra, we 
built a mathematical model describing HAS-7-HyWnt3 spatio-temporal dynamics as an extension of the classical activator-inhibitor models of Gierer and Meinhardt [30-32]. We used the framework of a hypothetical two-component activator-inhibitor concept based on current experimental evidence of HyWnt3 and beta-catenin/TCF dynamics (see Additional file 8: Supporting Information S1 for details of the model and its biological justification) and employ our experimental findings concerning HAS-7. In particular, we extend the previous models by explicitly distinguishing between betacatenin/TCF and HyWnt3 driven pattern formation. In addition, we verify how HAS-7 modulates formation of the body axes or the head in perturbed conditions (such as beta-catenin/TCF upregulation).

A scheme of the HAS-7-Wnt3 model is depicted in Fig. 5a. Based on our findings we postulate a negative feedback loop between HyWnt3 and HAS-7 (see Supporting Information for more details), representing one of the most frequent network motifs directing body-axis fine-scale segmentation in animals [33]. Additionally, we assume that HAS-7 expression is placed downstream of the organizer/HyWnt3, and thus it is only indirectly positively affected by beta-catenin/TCF. In particular, the HyWnt3-HAS7 interaction model comprises of two subsystems for organizer and axis formation, which act on different spatial scales. These include the small-scale HyWnt3-driven organizer pattern formation vs. beta-catenin/TCF signaling controlling large-scale body axis formation, as well as an additional subsystem directing tentacle formation (yellow blocks in Fig. 5a). The latter does not influence HAS-7-Wnt3 interactions. However, as it is affected by the body-axis and head system [30, 31], including it in the model makes possible additional model verification using, among other, data obtained under AZK treatment. 
As depicted in Fig. 5b, simulating the unperturbed system leads to the formation of a regular ring of tentacles below the hypostome. Furthermore, a single HyWnt3 expression spot appears at the tip of the hypostome and HAS-7 expression shows a distinct ring-like pattern. Similar patterns are observed in HAS-7 siRNA knockdown simulations without AZK treatment as modelling parameters were adjusted to preclude secondary axis formation in the unstimulated state (see SI file S1 for further details). Simulated AZK treatment assuming higher initial values of the source density [31] leads to multiple ectopic tentacles and elevated HAS-7 expression in the entire body column, but no additional axes (Fig. 5c). Combining the AZK treatment with HAS-7 depletion (Fig. 5d) indeed leads to loss of ectopic tentacles and the development of a secondary axis, whereas AZK treatment combined with HyDkk1/2/4 depletion leads to formation of a secondary organizer including the formation of ectopic tentacles (Fig. 5e). Finally, HyWnt3 overexpression (Fig. 5f) leads to a strong and body-wide expression of HAS-7. The only distinct difference between simulations and experimental observations is that a simulated HyWnt3 overexpression does not lead to a multiple axis phenotype but to a broadened Wnt3 expression domain at the head (Fig. 5f). The reason for this is that we describe each pattern formation system by the activator-inhibitor model, and an inherent mathematical property of this class of models does not allow changes in the activator production to cause an increase in the number of resulting heads (Marciniak-Czochra lab, in preparation). Thus, the applied activatorinhibitor model may be seen as an approximation of de novo pattern formation mechanism that is still unknown. In this context it is worth mentioning that de novo patterning may be based on not only chemical processes, but equally likely from mechanochemical, cellular and bio-electrical processes as well [34-36]. 
In summary, the proposed model qualitatively reproduces several of our experimental observations, including those which we did not even consider in the modelling process, such as the absence of ectopic tentacles in HAS-7-depleted animals treated with AZK. Analyzing the impact of model assumptions on the simulation results in the context of experimental data, we conclude that the function of HAS-7 is, most likely, to prevent the formation of multiple HyWnt3 spots. In particular, the dominance of a single organizer is ensured by HAS-7-driven degradation of the canonical Wnt ligands. We assume that this task is especially important when beta-catenin/TCF levels are increased, which may occur in Hydra under natural conditions, for example in interaction with the environment [37], or as a response to injury [29].

\section{Substrate specificity of the HAS-7 proteinase}

Altogether seven Wnt genes are expressed in the adult hypostome of Hydra (HyWnt1, $-3,-7,-9 / 10 a,-9 / 10 c,-11$, and -16) [7]. The size of their respective expression domains differs, with Wnt1, Wnt3, Wnt9/10a, c and Wnt11 showing a more confined spot-like expression reaching from the tip to the apical half of the hypostome, while Wnt7 and Wnt16 exhibit broader expression domains that extend to the tentacle bases. Wnt9/10b is not expressed in the adult polyp and Wnt5/8 are tentacle-specific [38]. Wnt2 is distinct in exhibiting a transitory expression at the site of bud initiation [7]. During head regeneration, the hypostomal Wnts are expressed in a temporally graded fashion with Wnt3, Wnt9/10c and Wnt11 being at the top of the cascade. Recombinant HyWnt3-His has been shown to induce head-forming capacity in tissue of the gastric region [7], indicating a primary role in initiating head formation. Our data indicate a redundant expression of several astacin proteinases in the sub-tentacle region with possibly 
similar substrate specificity. We have no evidence whether all head-specific Wnts are degraded by HAS-7 and closely related astacins, but considering that HyWnt3 acts at the top of the signaling cascade, it might serve as the primary substrate to inactivate the generation of a secondary organizer. When we screened the HyWnt3 amino acid sequence for putative cleavage sites for astacins, which were recently shown to have a strong preference for aspartate in the $\mathrm{P} 1$ ' position (first amino acid C-terminal of the cleavage site) and proline in P2' or P3' in substrate peptides [20], only D187 followed by Pro188 fulfilled this criterion in HyWnt3. To evaluate if this is supported by the structural properties of the two proteins we created a model of the HyWnt3:HAS-7 complex using X-ray crystal structures of Wnt8 [39], crayfish astacin [40, 41] and zebrafish hatching enzyme (ZHE-1) [42] as templates (Fig. 6a-b). The N-terminal prodomain, which confers protection of the active site is cleaved to transition the inactive zymogen into a catalytically potent astacin [22, 43, 44]. As a consequence, the alphahelical N-terminal domain of HyWnt3 is able to approach the active site cleft of HAS-7 opposite to the lipid-mediated Frizzled receptor binding site [39] (Fig. 6a). The KDP motif of the putative cleavage site in HyWnt3 is positioned where the pro-domain cleavage site would reside. It is embedded in the deep active-site cleft, which like a horizontal patch spans the entire catalytic domain (Fig. 6b). The catalytic zinc ion resides at the bottom of the active site cleft and shows a slightly distorted octahedral coordinating sphere complexed with the imidazole moieties of His132, His136 and His142, respectively. HyWnt3 binds to HAS-7 in an extended conformation and is anchored to the proteinase cleft in an antiparallel manner. Astacins are the only extracellular proteinases with a primary specificity for negatively charged residues (preferably aspartate) in the position C-terminally of the cleaved peptide bond [20] in the protein target and the elongated KKRK ${ }^{\star}$ DPRKIM motif would allow for most 
efficient cleavage. The negatively charged aspartate side chain at the P1' position is presumably bound by the positively charged arginine (R217) in the $S 1$ ' substrate binding site of HAS-7. In addition, a proline in close proximity in P2' as given in the HyWnt3 sequence is frequently observed in astacin substrates [20,45]. Mutation of the D187-P188 motif in HyWnt3 to alanine led to highly decreased secretion levels of the respective protein, indicative of protein instability or impaired folding in the secretory pathway, which precluded a meaningful cleavage assay.

Upon inspection of all Hydra Wnt sequences we found the DP motif at this position to be fully conserved only in HyWnt9/10a (Fig. 6c). Interestingly, non-canonical Wnts HyWnt5 and HyWnt8 [38] that are confined to the tentacle region and Wnts that have a broader expression domain in the head, like HyWnt7 and HyWnt16, were less conserved in this region and even lacked the aspartate residue. It is an attractive hypothesis that these Wnts act in confining the expression of HAS-7 and related astacins to the sub-tentacle region.

\section{Conclusions}

Metalloproteinases have a broad spectrum of activities and can be involved in extracellular matrix remodeling but also in processing of signaling molecules in a variety of developmental events. Here, we show that a member of the cnidarian astacin proteinase family acts as a negative feedback regulator of Wnt3 activity by direct morphogen processing, thereby providing a limitation of high canonical Wnt activity to the hypostomal organizer. Given the complex expression pattern of Wnt ligands in Hydra's head organizer, it will be interesting to unravel possible fine-tuned substrate specificities of the seemingly redundant astacin proteinase presence insulating the head region. 


\section{Methods}

Animals. Hydra vulgaris was used for all experiments. For siRNA experiments, a transgenic Hydra vulgaris strain expressing endodermal GFP and ectodermal RFP (reverse water melon) provided by Robert Steele's laboratory was used as described before [25]. The transgenic reporter line expressing eGFP under control of the Hydra Wnt3 promotor was described by Nakamura et al. [9]. The transgenic vector used to generate the strain expressing HyWnt3 under control of the actin promotor was produced by cloning the full-length HyWnt3 sequence between the actin promotor and actin 3' flanking sequences of the pBSSA-AR vector using Ncol and Xbal sites [9]. Generation of the transgenic actin::HyWnt3 line was performed by microinjection as described before [9]. All animals were maintained in artificial Hydra medium (HM, 1 $\mathrm{mM} \mathrm{CaCl}_{2}, 0.1 \mathrm{mM} \mathrm{MgCl}_{2}, 0.1 \mathrm{mM} \mathrm{KCl}_{1} \mathrm{mM} \mathrm{NaH}_{2} \mathrm{CO}_{3}, \mathrm{pH}$ 6.8) at $18^{\circ} \mathrm{C}$ in polystyrene dishes (Carl Roth) and fed two to three times per week with freshly hatched Artemia salina nauplii, unless indicated otherwise. Media was renewed 3-4 hrs after feeding and again the following day. Animals were starved for $24 \mathrm{hrs}$ prior to experiments, unless indicated otherwise.

Wnt proteolysis assays using tissue lysates. Hydra Wnt3 cDNA lacking the native leader peptide were subcloned into the pCEP-Pu mammalian expression vector, which introduces a BM-40 signal sequence and a C-terminal histidine tag as described previously [12]. Recombinant HyWnt3 was expressed using transiently or stably transfected HEK293T cells. For this, cells were seeded in full DMEM Medium (Gibco) in 6-well plates and transfected at $70 \%$ confluency using $1 \mu \mathrm{g}$ of the respective vector construct together with $5 \mu$ l transfection reagent (TransIT ${ }^{{ }_{-}} \mathrm{LT1}$ Reagent, Mirus Bio) in $200 \mu$ l serum-free DMEM medium after 20 min incubation time. The cells were 
maintained in a humidified $5 \% \mathrm{CO}_{2}$ atmosphere at $37^{\circ} \mathrm{C}$. After $48 \mathrm{hrs}$ the medium was harvested, followed by Ni-NTA sepharose (Qiagen) batch purification. For $1 \mathrm{ml}$ conditioned medium, $40 \mu \mathrm{l}$ Ni-NTA beads were added and incubated for $1 \mathrm{hr}$ at $4{ }^{\circ} \mathrm{C}$ under constant rotation. After a 2 min centrifugation step at $500 \mathrm{rpm}$ the beads were washed two times with PBS. Elution was performed with $250 \mathrm{mM}$ Imidazol for 15 min at RT. The beads were centrifuged at $500 \mathrm{rpm}$ and the supernatant containing the histidine-tagged Wnt protein was instantly frozen in liquid nitrogen and stored at -80 ${ }^{\circ} \mathrm{C}$. The eluted Hydra Wnt3 was verified by Western blotting using $10 \%$ SDS-PAGE gels. The proteins were transferred to PVDF membranes by wet blotting. Membranes were blocked for $1 \mathrm{hr}$ at RT in PBS containing 5\% BSA and 0.2\% Tween-20 (PBST), incubated with mouse Penta His antibody (Qiagen) at $1: 1000$ in $1 \%$ BSA at $4{ }^{\circ} \mathrm{C}$ overnight, washed $3 \times 5$ min with PBST and incubated with anti-mouse horseradish peroxidase-conjugated antibody at 1:10000 in PBST containing 5\% BSA for $1 \mathrm{hr}$ at RT. The membrane was washed $2 \times 5$ min with PBST and $2 \times 5$ min with PBS and blots were developed using a peroxidase substrate for enhanced chemiluminescence.

To generate Hydra body part lysates, 100 hydras were cut into four parts using a scalpel. Tentacles, head without tentacles, upper and lower gastric region were immediately transferred on ice, sonicated in $250 \mu \mathrm{l}$ ice cold PBS, centrifugated at $10000 \mathrm{rpm}$ for $1 \mathrm{~min}$ at $4{ }^{\circ} \mathrm{C}$, snap frozen and kept in aliquots at $-80{ }^{\circ} \mathrm{C}$. Body part lysate concentrations were adjusted to $4 \mathrm{mg} / \mathrm{ml}$ protein concentration using a nanodrop photometer and verified by Western blotting with alpha-tubulin antibody (SigmaAldrich). For the proteolysis assay, $\sim 10 \mathrm{ng}$ of purified HyWnt3-His was incubated with $15 \mu \mathrm{g}$ of the respective tissue lysates in a total volume of $12 \mu \mathrm{l}$ in PBS for $0,1,2,4,6$, 8 and 24 hrs at RT. Peak fractions from the ion exchange chromatography were incubated for $6 \mathrm{hrs}$. For inhibitor treatments, 1, 10-Phenanthroline (Sigma-Aldrich), 
Batimastat (Sigma-Aldrich) or EDTA (AppliChem) were added at $200 \mu \mathrm{M}$, respectively. Fetuin-B was diluted in PBS and incubated for the given time periods as indicated in Fig. 1f) The incubation was stopped by adding SDS-PAGE sample buffer (10\% glycerol, $50 \mathrm{mM}$ Tris- $\mathrm{HCl} \mathrm{pH} 6.8,0.02 \%$ bromophenol blue, $2 \%$ SDS, $1 \% 2$ mercaptoethanol) and heating at $96{ }^{\circ} \mathrm{C}$ for $5 \mathrm{~min}$. HyWnt3-His band intensities were documented by immunoblotting as described above. Unspecific proteolysis in HL was monitored adding $15 \mu \mathrm{g}$ of the lysate to $1 \mu \mathrm{g}$ of BSA (Roth) in a total volume of $12 \mu \mathrm{l}$ in PBS and incubation at RT. The reaction was stopped by adding sample buffer and heat denaturation at $96{ }^{\circ} \mathrm{C}$ for 5 min after the same time periods as for HyWnt3-His. Samples were analyzed using 10\% SDS-PAGE gels and Coomassie staining.

Full-length recombinant HyDkk1/2/4-His protein was expressed in E. coli BL21 (DE3) cells from a pET15b vector (Novagen) and purified under native conditions from the supernatant of cell pellets lysed by several freeze/thaw steps in PBS. The cleared supernatant was filtered and purified using Ni-NTA agarose beads (Qiagen). The eluted protein solution was dialyzed against PBS and purity was checked by SDSPAGE. For the proteolysis assay, $10 \mathrm{ng}$ of purified HyDkk/1/2/4-His was incubated with $15 \mu \mathrm{g}$ of the respective tissue lysates in a total volume of $12 \mu \mathrm{l}$ in PBS for $0,1,2,4,6$, 8 and 24 hrs at RT. HyDkk1/2/4-His band intensities were documented by immunoblotting as described above.

The sequence for the Hydra Cadherin CAD1-2 domain (15-239) was amplified by PCR using a large 5' cDNA fragment of Hydra Cadherin as template. The PCR product was cloned into the pET19B vector (Novagen) using Ndel and BamHI sites and the recombinant protein was expressed in E. coli BL21 (DE3) cells. Inclusion bodies were solubilized in PBS, $8 \mathrm{M}$ urea and bound to Ni-NTA agarose beads. Refolding was performed by changing to PBS buffer prior to elution with PBS, 250mM imidazole, 0.4 
M L-arginine (AppliChem). The eluted protein solution was dialyzed against PBS and purity was checked by SDS-PAGE. $10 \mathrm{ng}$ of the purified protein was used as substrate with $5 \mu \mathrm{l}$ of either the HyWnt3(+) and HyWnt3(-) HL fraction. Detection of the Hydra cadherin fragment by immunoblotting was performed as described above.

The polyclonal HAS-7 specific antibody was raised against a peptide corresponding to a sequence between the catalytic and ShKT domains (CKGGGNPPTGPPTAPP). For Western blotting, a PVDF membrane was blocked in 5\% skimmed milk powder in PBS, $0.2 \%$ Tween-20, for $1 \mathrm{hr}$ at RT. The primary antibody was applied at $1: 1000$ in $1 \%$ skimmed milk powder in PBST and membranes were incubated overnight at $4{ }^{\circ} \mathrm{C}$. A peroxidase-conjugated anti-rabbit antibody (Jackson ImmunoResearch Laboratories, Inc.) was applied in blocking solution at 1:10000 for $1 \mathrm{hr}$ at RT. Quantification of Western blot band intensities was performed using ImageStudioLite software.

Ion exchange chromatography and mass spectrometry analysis. A pool of head lysate was generated by decapitating 400 animals closely beneath the head region and removing tentacles using a scalpel. Head tissue pieces were resuspended in ice cold $20 \mathrm{mM}$ Tris- $\mathrm{HCl}, \mathrm{pH} 7.5$, and lysed on ice by passing at least 10 times through a 20-gauge needle. The lysate was centrifuged for $15 \mathrm{~min}(14000 \mathrm{rpm})$ at $4{ }^{\circ} \mathrm{C}$ and the supernatant was applied to anion exchange chromatography using a MonoQ HR5/5 column (GE healthcare). The eluent buffer contained $1 \mathrm{M} \mathrm{NaCl}$ in $20 \mathrm{mM}$ Tris- $\mathrm{HCl}, \mathrm{pH}$ 7.5. Peak fractions indicated in Additional file 1: Fig. S1 were screened for HyWnt3His proteolytic activity as described above and frozen at $-20^{\circ} \mathrm{C}$ until further use. Pooled HyWnt3(+) and HyWnt3(-) fractions were subjected to mass spectrometry analysis after in solution tryptic digestion. Peptide separation was achieved using a nano Acquity UPLC system (Waters). Protein mass spectrometry analyses were performed 
as previously described [46]. In short: The nano UPLC system was coupled online to an LTQ OrbitrapXL mass spectrometer (Thermo Fisher Scientific). Data dependent acquisition with Xcalibur 2.0.6 (Thermo Fisher Scientific) was performed by one FTMS scan with a resolution of 60000 and a range from 370 to $2000 \mathrm{~m} / \mathrm{z}$ in parallel with six MS/MS scans of the most intense precursor ions in the ion trap. The mgf files processed from the MS raw files were used for protein database searches with the MASCOT search engine (Matrix Science, version 2.2) against NCBI GenBank Proteins (version of October 28, 2015). Domain composition of GenBank protein accessions was analyzed against CDD (NCBI) and InterProScan 5 (EMBL-EBI).

\section{Expression and purification of recombinant HAS-7. Expression of HAS-7 occurred} in insect cells using the Bac-to-Bac system (Invitrogen, Thermo Fisher Scientific). A synthetic fragment of the HAS-7 full-length cDNA (Biomatik) was subcloned into the pCEP-Pu mammalian expression vector, which introduces a C-terminal histidine tag and the fragment containing the $\mathrm{C}$-terminal $\mathrm{His}_{(6)}$-tag was inserted into the donor plasmid pFASTBac1 to generate bacmids in E. coli DH10Bac cells for transfection of insect cells according to the manufacturer's manual. Amplification of recombinant baculoviruses occurred in Spodoptera frugiperda 9 cells (Sf9 CCLV-RIE 203, Friedrich-Löffler Institute, Greifswald, Germany) growing as adherent monolayer in Grace's insect medium supplemented with $100 \mathrm{U} / \mathrm{ml}$ penicillin and $100 \mu \mathrm{g} / \mathrm{ml}$ streptomycin. For expression of HAS-7-His(6) Trichoplusia ni cells (BTI-TN-5B1-4/High Five-cells CCLV-RIE 305, Friedrich-Löffler Institute, Greifswald, Germany) were cultured as suspension in Express Five SFM containing $100 \mathrm{U} / \mathrm{ml}$ penicillin, $100 \mu \mathrm{g} / \mathrm{ml}$ streptomycin and $16.4 \mathrm{mM} \mathrm{L-Glutamine.} \mathrm{Infected} \mathrm{High} \mathrm{Five} \mathrm{cells} \mathrm{were} \mathrm{incubated} \mathrm{for}$ 72 hrs at $27^{\circ} \mathrm{C}$ in Fernbach flasks (shaking incubator Multitron, INFORS). Following 
centrifugation, proteins were precipitated at $10{ }^{\circ} \mathrm{C}$ by step wise addition of solid ammonium sulfate to the supernatant resulting in a $60 \%$ ammonium sulfate saturation. After further stirring over night at $10^{\circ} \mathrm{C}$ and centrifugation $\left(9000 \times \mathrm{g}, 90 \mathrm{~min}, 10^{\circ} \mathrm{C}\right)$ the harvested pellet was resuspended in $1 / 10$ of the expression volume in $50 \mathrm{mM}$ Tris$\mathrm{HCl} \mathrm{pH}$ 7.6, $300 \mathrm{mM} \mathrm{NaCl}, 20 \mathrm{mM}$ imidazole and dialyzed against the same buffer. The cleared supernatant gained after centrifugation $\left(8000 \times \mathrm{g}, 10 \mathrm{~min}, 4^{\circ} \mathrm{C}\right.$ ) was applicated onto a Ni-NTA column (Qiagen). Following several washing steps with 50 $\mathrm{mM}$ Tris- $\mathrm{HCl} \mathrm{pH} 7.6,300 \mathrm{mM} \mathrm{NaCl}$ containing increasing imidazole concentrations (20 $\mathrm{mM}, 50 \mathrm{mM}$ and $100 \mathrm{mM}$ ), the protein was finally eluted in buffer containing $250 \mathrm{mM}$ imidazole. The elution fractions were pooled, dialyzed against PBS (140 mM NaCl, 2.7 $\mathrm{mM} \mathrm{KCl}, 10 \mathrm{mM} \mathrm{Na}_{2} \mathrm{HPO}_{4} 2 \mathrm{H}_{2} \mathrm{O}, 1.8 \mathrm{mM} \mathrm{KH}_{2} \mathrm{PO}_{4}, \mathrm{pH} 7.4$ ) and concentrated (Millipore Amicon Ultra, $3 \mathrm{~K}$ ). SDS-PAGE and transfer to PVDF was performed as described previously [47]. The membrane was blocked in 3\% BSA in TBS for $2 \mathrm{hrs}$, incubated for $1 \mathrm{hr}$ with Penta-His antibody at 1:2000 and further incubated for $1 \mathrm{hr}$ with secondary antibody (goat anti mouse POX 1:7500 in 7.5\% skimmed milk powder in TBS). After each antibody treatment three washing steps were inserted ( 2 x TBST, $1 \times$ TBS). The Clarity Western ECL Substrate (Biorad) was used for detection.

In situ hybridization. Customized LNA digoxygenin-labeled RNA probes were designed and produced by Qiagen corresponding to the antisense strands of the respective astacin cDNAs (sequences are given in Additional file 6: Table S3). The whole-mount ISH procedure was performed as described previously [48]. For hybridization, the LNA probe was added to a final concentration of $1 \mu \mathrm{M}$ in fresh hybridization solution (1:1 mixture of deionized formamide and buffer containing $5 x$ SSC (750 mM NaCl, $75 \mathrm{mM}$ sodium citrate), $0.2 \mathrm{mg} / \mathrm{ml}$ yeast tRNA, $2 \%$ of $50 \mathrm{x}$ 
Denhardt's solution, $0.1 \mathrm{mg} / \mathrm{ml}$ heparin, $0.1 \%$ Tween-20 and $0.1 \%$ CHAPS) and hybridized for $\sim 60 \mathrm{hrs}$ at $55^{\circ} \mathrm{C}$. Digoxygenin-labelled RNA probes for HAS-1, HAS-7 and HMP-1 corresponding to the sense and antisense strands were prepared using an RNA labelling in vitro transcription kit (Roche). ISH probe sequences covered the predicted full-length mRNA sequence for each gene. The further procedure was as described previously [12]. Samples were finally mounted in $90 \%$ glycerol in PBS or in Mowiol 4-88 (Carl Roth) and images were acquired with a Nikon Digital Sight DS-U1 camera mounted on Nikon Eclipse 80i and imaging software NIS Elements (3.10, SP3, Hotfix, Build645). Further image processing was performed with Adobe Photoshop CS6 and Fiji.

Alsterpaullone and iCRT14 treatment for ISH analysis. 80 budless hydras, which were fed the day before, were incubated in $5 \mu \mathrm{M}$ ALP (Sigma-Aldrich) in DMSO in 100 $\mathrm{ml} \mathrm{HM}$. The animals were kept in the dark at $18{ }^{\circ} \mathrm{C}$ for $24 \mathrm{hrs}$. After incubation, the HM was changed every day. Samples were taken for ISH at $24 \mathrm{hrs}, 48 \mathrm{hrs}$ and $72 \mathrm{hrs}$ after ALP treatment. Hydras incubated for $24 \mathrm{hrs}$ in DMSO and fixed after $72 \mathrm{hrs} \mathrm{served}$ as control. For the $\beta$-catenin inhibitor experiment, 30 budless hydras, which were fed the day before, were collected in $10 \mathrm{ml}$ petri dishes filled with HM. iCRT14 (SigmaAldrich) in DMSO was added to the petri dishes at a final concentration of $10 \mu \mathrm{M}$. The petri dishes were incubated in the dark at $18^{\circ} \mathrm{C}$. After $48 \mathrm{hrs}$ samples were taken for WISH.

Electroporation with siRNAs. siRNAs (HPLC grade) (siGFP, scrambled siGFP, siHAS-7, siHMP1, siHyDkk1/2/4, siHyWnt3, see Additional file 7: Table S4 for sequences) were purchased from Qiagen. Electroporation of siRNA was performed as 
described recently [12] using $3 \mu \mathrm{M}$ of siGFP (1 $\mu \mathrm{M}$ siGFP and $2 \mu \mathrm{M}$ scrambled siGFP) or a combination of siGFP and target siRNAs ( $1 \mu \mathrm{M}$ each). Immediately after the pulse, $500 \mu$ l restoration medium consisting of $80 \% \mathrm{HM}$ and $20 \%$ hyperosmotic dissociation medium $\left(6 \mathrm{mM} \mathrm{CaCl}_{2}, 1.2 \mathrm{mM} \mathrm{MgSO} 4,3.6 \mathrm{mM} \mathrm{KCl}, 12.5 \mathrm{mM}\right.$ TES, $6 \mathrm{mM}$ sodium pyruvate, $6 \mathrm{mM}$ sodium citrate, $6 \mathrm{mM}$ glucose and $50 \mathrm{mg} / \mathrm{l}$ rifampicin, $100 \mathrm{mg} / \mathrm{l}$ streptomycin, $50 \mathrm{mg} / \mathrm{l}$ kanamycin, $\mathrm{pH}$ 6.9) was added to the cuvette, the animals were transferred to Petri dishes containing restoration medium and allowed to recover for one day. Viable polyps were transferred to new dishes containing $\mathrm{HM}$ and maintained under standard culture conditions. For AZK treatment, animals were incubated at 6 days post-electroporation either with $0.1 \%$ DMSO or $50 \mathrm{nM} \mathrm{AZK}$ in HM for $16 \mathrm{hrs}$. Thereafter, they were rinsed in HM several times and cultured under standard conditions. 5 days after AZK treatment, the animals were anesthetized in $1 \mathrm{mM}$ Linalool (Sigma-Aldrich) [49] using a Nikon SMZ25 stereomicroscope equipped with Nikon DSRi2 high-definition color camera. Note that AZK instead of ALP was used in siRNA experiments to be able to reduce the total inhibitor concentration applied on the electroporated animals. $50 \mathrm{nM} \mathrm{AZK}$ induces the same grade of ectopic tentacles as 5 $\mu \mathrm{M}$ ALP when applied in the standard assay published previously [38].

Chromatin immunoprecipitation. Chromatin immunoprecipitation analysis was carried out as described recently by using sheared extracts from formaldehyde-treated Hydra animals, which have been treated without or with $5 \mu \mathrm{M}$ ALP (48hrs) and an antiserum directed against a recombinant Hydra TCF protein [12, 29]. PCR of precipitated DNA was done using specific primers flanking the potential TCF binding sites in the 5'-regulatory regions of the Hydra HAS-7 or the TSP gene (Fig. 4g-h). PCR primer sequences: TCF binding motif in HAS-7 (5'-GCTGTTATCTGTCCGCTTTC- 
3'/5'-CCATATAGAGGCCACACACC-3'), and the proximal TCF binding motif in TSP (5'-TTGAAGGCATTTAACAACTTGC-3'/5'-TGCCCAAATGTAAAGTTCTGTG-3').

Real-time Quantitative PCR. The RNA isolation was performed as described previously [46]. 60 hydra heads per condition were isolated either from steady state AEP polyps or siRNA treated transgenic reverse water melon animals by removing the tentacles and gastric region using a scalpel. The heads were immediately transferred into $200 \mu \mathrm{l} \mathrm{TRIzol}$ and stored at $-20^{\circ} \mathrm{C}$. For siRNA (siGFP or siHAS-7) treated hydras, head samples were isolated six days after electroporation. cDNA synthesis was performed using the SensiFAST cDNA Synthesis Kit (Bioline) according to manufacturer's instructions. RT-qPCR was carried out with a StepOnePlus тм instrument (Applied Biosystems, Thermo Fisher Scientific) using the SensiFAST SYBR-Hi-ROX Kit (Bioline) according to the manufacturer's instructions. The transcript level analysis was done by the $\Delta \Delta \mathrm{C}(\mathrm{T})$-Method with Elongation Factor 1-a (EF-1a) as a house keeping gene for normalization. Three biological replicates were performed for each experiment and triplicate measurements were made for each sample in each experiment. No template conditions served as negative controls. The data are presented as relative quantity $(R Q)$ by $2^{\wedge}(-\Delta \Delta C(T))$ calculation. qPCR primer sequences are given in Additional file 7: Table S4.

Xenopus experiments. In vitro fertilization, embryo culture and culture of explants were carried out as described [50]. Staging was done according to Nieuwkopp [51]. mRNA was produced with the mMessage mMachine SP6 trancription Kit (Ambion) from the HyWnt3, XWnt8, HAS-7, flag-tagged GFP (control mRNA) ORFs in the respective linearized pCS2+ vectors. mRNA was purified with a phenol/chloroform 
extraction and a subsequent isopropanol precipitation. Injections were done into the marginal zone of both ventral cells of the 4-cell stage. Total amounts of each injected mRNA were: XWnt8 (10 pg), HyWnt3 (10 or 100 pg), HyWnt3/HAS-7 (100 pg each), GFP control (100 pg).

Structural modeling. Protein-Protein docking experiments were performed in ClusPro2 [52] using the crystal structures of Xenopus Wnt8 (pdb-code:4F0A) and promeprin B (pdb-code: 4GWM). The propetide E25-G66 blocking access to the active site cleft and disordered loop segments in Wnt8 were removed to allow for proper structural analysis. Targeted search matrices were chosen by selecting the zincbinding site of promeprin $B(\mathrm{H} 132-\mathrm{H} 136-\mathrm{H} 142)$ and the putative cleavage site of HyWnt3 (K186-D187-P188) as attractive search targets. Based on Lennart-Jones potentials, distance metrices and electrostatic evaluations the best 100 docking hits were subject to gradient energy minimization in the Crystallography and NMR system. 14 The lowest energy structures were further subject to 500 cycles of unrestrained Powell minimization. Harmonic restraints were imposed on the target molecule (2 $\left.\mathrm{kcal} / \mathrm{mol} \AA^{2}\right)$ with increased weight $\left(25 \mathrm{kcal} / \mathrm{mol} \AA^{2}\right)$. Protein structure and model assessment tools were used to verify the quality of the modeled structure. Additionally, HAS-7 modelling was performed using Modeller [53] implemented in Chimera [54] with astacin complexed to a transition state analogue inhibitor (pdb-code: 1QJI) and zebrafisch hatching enzyme (3LQB), the latter being the most closely HAS-7-related astacin with known structure to date. 


\section{List of abbreviations}

ALP: Alsterpaullone; AZK: 1-Azakenpaullone; BMP: bone morphogenetic protein; ChIP: chromatin immunoprecipitation; DFG: Deutsche Forschungsgemeinschaft; Dkk: Dickkopf; DMEM: Dulbecco's modified Eagle's medium; DMSO: dimethylsulfoxid; FTMS: Fourier transform mass spectrometry; GFP: Green fluorescent protein; GSK3: glycogen synthase kinase 3; HAS: Hydra astacin; HPLC: high performance liquid chromatography; HL: head lysate; Hm: Hydra magnipapillata; HM: Hydra medium; HMP: Hydra metalloproteinase; iCRT: inhibitor of $\beta$-catenin-responsive transcription; ISH: in situ hybridization; LNA: locked nucleic acid; ORF: open reading frame; PBS: phosphate-buffered saline; PCR: polymerase chain reaction, PDB: protein data bank; RFP: red fluorescent protein, RT: room temperature; SEC: Secretome; ShkT: Stichodactyla toxin; siRNA: short interfering RNA; TCF: T-cell factor; TES: 2-[Tris(hydroxymethyl)methylamino]-1-ethane sulfonic acid; TSP: Thrombospondin; Wnt: wingless/integrated; UPLC: ultra-high-performance liquid chromatography; WISH: whole mount in situ hybridization; $\mathrm{X}$ : Xenopus. 


\section{Declarations}

\section{Ethics approval and consent to participate}

Xenopus experiments were approved by the state review board of BadenWuerttemberg, Germany (License number: G-13/186) and performed according to the federal and institutional guideline.

\section{Competing interests}

The authors declare that they have no competing interests.

\section{Funding}

This work was supported by grants of the German Science Foundation (OE 416/7-1 to S.Ö., SFB1324/B06 to A.M-C., and FOR 1036/A01 and SFB1324/A05 to T.W.H). J.S. is a Tier-1 CRC in Structural Biology and Biophysics. This research project was funded by the Canadian Institute of Health and Research (CIHR-201610PJT-152935). This work was supported by JGU Research Funds to I.Y. and W.S.

\section{Authors' contributions}

SÖ, BZ, WS and IY designed experiments. BZ, BT, SK, MF, SK and IY performed experiments. JS and WS performed the structural modeling. UW and MS performed the mass spectrometry analysis. MH designed and performed the ChIP analysis. TWH provided the HyWnt3 transgenes. $\mathrm{MM}$ and $\mathrm{AMC}$ performed the mathematical modeling. SÖ, BZ, MM, WS and TWH wrote the manuscript. All authors read and approved the final manuscript. 


\section{Acknowledgements}

We thank Yukio Nakamura for the preparation of the actin::HyWnt3 pBSSA-AR vector, Jutta Tennigkeit for preparation of the recombinant expression constructs, Ann-Kathrin Heilig for performing in situ hybridization experiments, Alexander Hirth and Christof Niehrs for sharing Xenopus embryos and assisting with mRNA injections. We thank Kane Puglisi for technical assistance in the ChIP analysis.

\section{References}

1. De Robertis EM: Spemann's organizer and the self-regulation of embryonic fields. Mech Dev 2009, 126(11-12):925-941.

2. Niehrs C: On growth and form: a Cartesian coordinate system of Wnt and BMP signaling specifies bilaterian body axes. Development 2010, 137(6):845-857.

3. Petersen CP, Reddien PW: Wnt signaling and the polarity of the primary body axis. Cell 2009, 139(6):1056-1068.

4. Broun M, Gee L, Reinhardt B, Bode HR: Formation of the head organizer in hydra involves the canonical Wnt pathway. Development 2005, 132(12):2907-2916.

5. Gee L, Hartig J, Law L, Wittlieb J, Khalturin K, Bosch TC, Bode HR: betacatenin plays a central role in setting up the head organizer in hydra. Dev Biol, 340(1):116-124.

6. Hobmayer B, Rentzsch F, Kuhn K, Happel CM, von Laue CC, Snyder P, Rothbacher U, Holstein TW: WNT signalling molecules act in axis formation in the diploblastic metazoan Hydra. Nature 2000, 407(6801):186-189.

7. Lengfeld T, Watanabe H, Simakov O, Lindgens D, Gee L, Law L, Schmidt HA, Ozbek S, Bode $\mathrm{H}$, Holstein TW: Multiple Wnts are involved in Hydra organizer formation and regeneration. Dev Biol 2009, 330(1):186-199.

8. Broun M, Bode HR: Characterization of the head organizer in hydra. Development 2002, 129(4):875-884.

9. Nakamura Y, Tsiairis CD, Ozbek S, Holstein TW: Autoregulatory and repressive inputs localize Hydra Wnt3 to the head organizer. Proc Natl Acad Sci U S A 2011, 108(22):9137-9142.

10. Vogg MC, Beccari L, Iglesias Olle L, Rampon C, Vriz S, Perruchoud C, Wenger Y, Galliot B: An evolutionarily-conserved Wnt3/beta-catenin/Sp5 feedback loop restricts head organizer activity in Hydra. Nat Commun 2019, 10(1):312.

11. Guder C, Pinho S, Nacak TG, Schmidt HA, Hobmayer B, Niehrs C, Holstein TW: An ancient Wnt-Dickkopf antagonism in Hydra. Development 2006, 133(5):901-911.

12. Lommel M, Strompen J, Hellewell AL, Balasubramanian GP, Christofidou ED, Thomson AR, Boyle AL, Woolfson DN, Puglisi K, Hartl M et al: Hydra Mesoglea 
Proteome Identifies Thrombospondin as a Conserved Component Active in Head Organizer Restriction. Sci Rep 2018, 8(1):11753.

13. Piccolo S, Agius E, Lu B, Goodman S, Dale L, De Robertis EM: Cleavage of Chordin by Xolloid metalloprotease suggests a role for proteolytic processing in the regulation of Spemann organizer activity. Cell 1997, 91(3):407-416.

14. Zhang X, Abreu JG, Yokota C, MacDonald BT, Singh S, Coburn KL, Cheong $\mathrm{SM}$, Zhang MM, Ye QZ, Hang $\mathrm{HC}$ et al: Tiki1 is required for head formation via Wnt cleavage-oxidation and inactivation. Cell 2012, 149(7):1565-1577.

15. Yan L, Fei K, Zhang J, Dexter S, Sarras MP, Jr.: Identification and characterization of hydra metalloproteinase 2 (HMP2): a meprin-like astacin metalloproteinase that functions in foot morphogenesis. Development 2000, 127(1):129-141.

16. Yan L, Leontovich A, Fei K, Sarras MP, Jr.: Hydra metalloproteinase 1: a secreted astacin metalloproteinase whose apical axis expression is differentially regulated during head regeneration. Dev Biol 2000, 219(1):115-128.

17. Yan L, Pollock GH, Nagase H, Sarras MP, Jr.: A $25.7 \times 10(3)$ M(r) hydra metalloproteinase (HMP1), a member of the astacin family, localizes to the extracellular matrix of Hydra vulgaris in a head-specific manner and has a developmental function. Development 1995, 121(6):1591-1602.

18. Chapman JA, Kirkness EF, Simakov O, Hampson SE, Mitros T, Weinmaier T, Rattei T, Balasubramanian PG, Borman J, Busam D et al: The dynamic genome of Hydra. Nature 2010, 464(592):7288.

19. Karmilin K, Schmitz C, Kuske M, Korschgen H, Olf M, Meyer K, Hildebrand A, Felten M, Fridrich $S$, Yiallouros I et al: Mammalian plasma fetuin-B is a selective inhibitor of ovastacin and meprin metalloproteinases. Scientific Reports 2019, 9.

20. Becker-Pauly C, Barre O, Schilling O, Auf dem Keller U, Ohler A, Broder C, Schutte A, Kappelhoff R, Stocker W, Overall CM: Proteomic analyses reveal an acidic prime side specificity for the astacin metalloprotease family reflected by physiological substrates. Mol Cell Proteomics 2011, 10(9):M111 009233.

21. Rholam M, Brakch N, Germain D, Thomas DY, Fahy C, Boussetta H, Boileau $G$, Cohen $P$ : Role of amino acid sequences flanking dibasic cleavage sites in precursor proteolytic processing. The importance of the first residue Cterminal of the cleavage site. Eur J Biochem 1995, 227(3):707-714.

22. Gomis-Ruth FX, Trillo-Muyo S, Stocker W: Functional and structural insights into astacin metallopeptidases. Biological Chemistry 2012, 393(10):10271041.

23. Schmidt T, David CN: Gland-Cells in Hydra - Cell-Cycle Kinetics and Development. Journal of Cell Science 1986, 85:197-215.

24. Siebert S, Farrell JA, Cazet JF, Abeykoon Y, Primack AS, Schnitzler CE, Juliano CE: Stem cell differentiation trajectories in Hydra resolved at single-cell resolution. Science 2019, 365(6451): eaav9314.

25. Lommel M, Tursch A, Rustarazo-Calvo L, Trageser B, Holstein TW: Genetic knockdown and knockout approaches in Hydra. bioRxiv 2017. 
26. Shoemark DK, Ziegler B, Watanabe H, Strompen J, Tucker RP, Ozbek S, Adams JC: Emergence of a Thrombospondin Superfamily at the Origin of Metazoans. Mol Biol Evol 2019, 36(6):1220-1238.

27. Schlenzig D, Wermann M, Ramsbeck D, Moenke-Wedler T, Schilling S: Expression, purification and initial characterization of human meprin beta from Pichia pastoris. Protein Expr Purif 2015, 116:75-81.

28. Watanabe H, Schmidt HA, Kuhn A, Hoger SK, Kocagoz Y, Laumann-Lipp N, Ozbek S, Holstein TW: Nodal signalling determines biradial asymmetry in Hydra. Nature 2014, 515(7525):112-5.

29. Gufler S, Artes B, Bielen H, Krainer I, Eder MK, Falschlunger J, Bollmann A, Ostermann $\mathrm{T}$, Valovka $\mathrm{T}$, Hartl $\mathrm{M}$ et al: beta-Catenin acts in a positionindependent regeneration response in the simple eumetazoan Hydra. Dev Biol 2018, 433(2):310-323.

30. Meinhardt $\mathrm{H}$ : A model for pattern formation of hypostome, tentacles, and foot in hydra: how to form structures close to each other, how to form them at a distance. Developmental biology 1993, 157(2):321-333.

31. Meinhardt H: Turing's theory of morphogenesis of 1952 and the subsequent discovery of the crucial role of local self-enhancement and long-range inhibition. Interface Focus 2012, 2(4):407-416.

32. Meinhardt $\mathrm{H}$, Gierer A: Pattern formation by local self-activation and lateral inhibition. Bioessays 2000, 22(8):753-760.

33. Briscoe J, Small S: Morphogen rules: design principles of gradientmediated embryo patterning. Development (Cambridge, England) 2015, 142:3996-4009.

34. Hiscock TW, Megason SG: Mathematically guided approaches to distinguish models of periodic patterning. Development 2015, 142(3):409419.

35. Gierer A, Meinhardt $\mathrm{H}$ : A theory of biological pattern formation. Kybernetik 1972, 12(1):30-39.

36. Pietak A, Levin M: Bioelectric gene and reaction networks: computational modelling of genetic, biochemical and bioelectrical dynamics in pattern regulation. Journal of The Royal Society Interface 2017, 14(134):20170425.

37. Taubenheim J, Willoweit-Ohl D, Knop M, Franzenburg S, Bosch TCG, Fraune $\mathrm{S}$ : Bacteria-and temperature-regulated peptides modulate beta-catenin signaling in Hydra. bioRxiv 2019:747303.

38. Philipp I, Aufschnaiter R, Ozbek S, Pontasch S, Jenewein M, Watanabe H, Rentzsch F, Holstein TW, Hobmayer B: Wnt/beta-catenin and noncanonical Wnt signaling interact in tissue evagination in the simple eumetazoan Hydra. Proc Natl Acad Sci U S A 2009, 106(11):4290-4295.

39. Janda CY, Waghray D, Levin AM, Thomas C, Garcia KC: Structural Basis of Wnt Recognition by Frizzled. Science 2012, 337(6090): 59-64.

40. Bode W, Gomis-Ruth FX, Huber R, Zwilling R, Stocker W: Structure of astacin and implications for activation of astacins and zinc-ligation of collagenases. Nature 1992, 358(6382):164-167.

41. Gomis-Ruth FX, Stocker W, Huber R, Zwilling R, Bode W: Refined 1.8 A X-ray crystal structure of astacin, a zinc-endopeptidase from the crayfish Astacus astacus L. Structure determination, refinement, molecular structure and comparison with thermolysin. J Mol Biol 1993, 229(4):945968. 
42. Okada A, Sano K, Nagata K, Yesumasu S, Ohtsuka J, Yamamura A, Kubota K, luchi I, Tanokura M: Crystal Structure of Zebrafish Hatching Enzyme 1 from the Zebrafish Danio rerio. J Mol Biol 2010, 402(5):865-878.

43. Guevara T, Yiallouros I, Kappelhoff R, Bissdorf S, Stocker W, Gomis-Ruth FX: Proenzyme structure and activation of astacin metallopeptidase. $J$ Biol Chem 2010, 285(18):13958-13965.

44. Yiallouros I, Kappelhoff R, Schilling O, Wegmann F, Helms MW, Auge A, Brachtendorf G, Berkhoff EG, Beermann B, Hinz HJ et al: Activation mechanism of pro-astacin: role of the pro-peptide, tryptic and autoproteolytic cleavage and importance of precise amino-terminal processing. J Mol Biol 2002, 324(2):237-246.

45. Stocker W, Ng M, Auld DS: Fluorescent oligopeptide substrates for kinetic characterization of the specificity of Astacus protease. Biochemistry 1990, 29(45):10418-10425.

46. Petersen HO, Hoger SK, Looso M, Lengfeld T, Kuhn A, Warnken U, NishimiyaFujisawa C, Schnolzer M, Kruger M, Ozbek S et al: A Comprehensive Transcriptomic and Proteomic Analysis of Hydra Head Regeneration. Mol Biol Evol 2015, 32(8):1928-47.

47. Korschgen H, Kuske M, Karmilin K, Yiallouros I, Balbach M, Floehr J, Wachten $D$, Jahnen-Dechent W, Stocker W: Intracellular activation of ovastacin mediates pre-fertilization hardening of the zona pellucida. Mol Hum Reprod 2017, 23(9):607-616.

48. Schuler A, Schmitz G, Reft A, Ozbek S, Thurm U, Bornberg-Bauer E: The rise and fall of TRP-N, an ancient family of mechanogated ion channels, in Metazoa. Genome Biol Evol 2015, 7(6):1713-27.

49. Goel T, Wang R, Martin S, Lanphear E, Collins ES: Linalool acts as a fast and reversible anesthetic in Hydra. PLoS One 2019, 14(10):e0224221.

50. Kumar S, Zigman M, Patel TR, Trageser B, Gross JC, Rahm K, Boutros M, Gradl D, Steinbeisser H, Holstein T et al: Molecular dissection of Wnt3aFrizzled8 interaction reveals essential and modulatory determinants of Wnt signaling activity. BMC Biol 2014, 12:44.

51. Nieuwkoop PD: Normal table of Xenopus laevis (Daudin) : a systematical and chronological survey of the development from the fertilized egg till the end of metamorphosis, 2nd edn. Amsterdam: North-Holland Publ. Company; 1967.

52. Kozakov D, Hall DR, Xia B, Porter KA, Padhorny D, Yueh C, Beglov D, Vajda S: The ClusPro web server for protein-protein docking. Nat Protoc 2017, 12(2):255-278.

53. Sali A, Blundell TL: Comparative Protein Modeling by Satisfaction of Spatial Restraints. J Mol Biol 1993, 234(3):779-815.

54. Pettersen EF, Goddard TD, Huang CC, Couch GS, Greenblatt DM, Meng EC, Ferrin TE: UCSF Chimera--a visualization system for exploratory research and analysis. J Comput Chem 2004, 25(13):1605-1612. 


\section{Figures}

Fig. 1
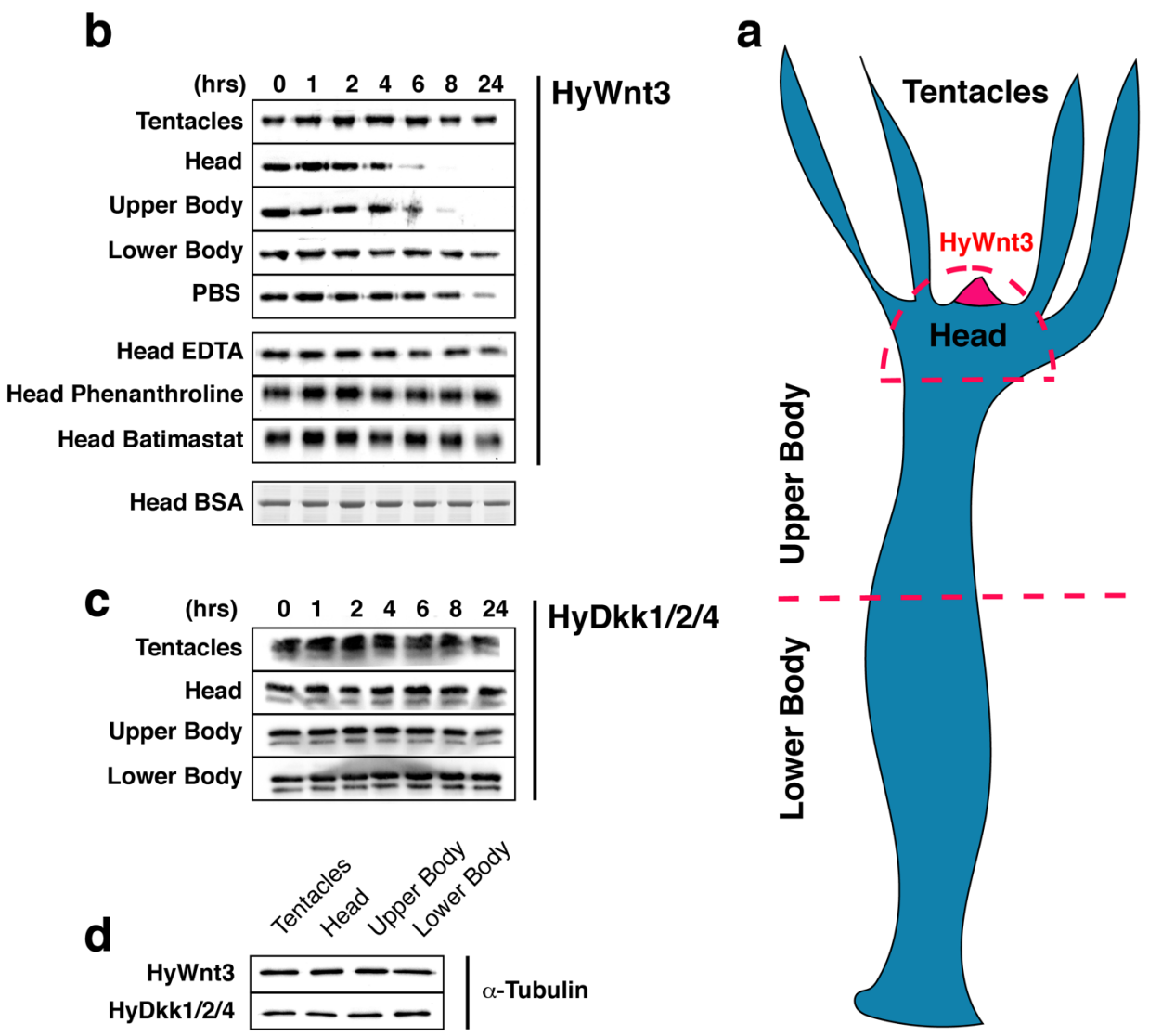

e HyWnt3(+)

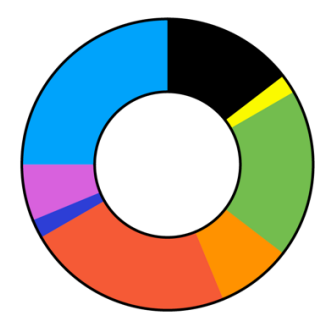

HyWnt3(-)

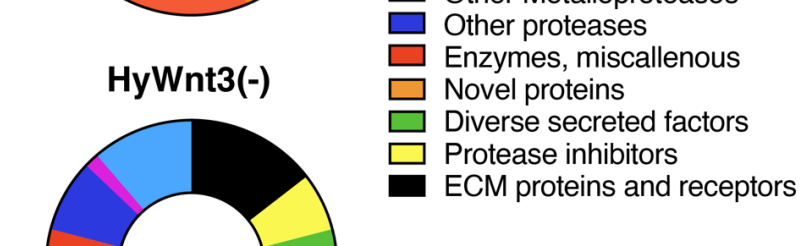

f

Head lysate

(hrs) $\begin{array}{llllllll}0 & 1 & 2 & 4 & 6 & 8 & 24 & \text { Fetuin B }\end{array}$

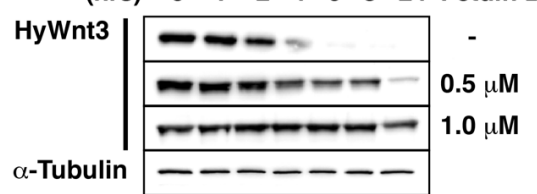


Fig. 1. Screen for HyWnt3 proteolytic activity in Hydra tissue lysates. (a) Schematic representation of body plan. Body parts used for lysates in b-d are indicated. The hypostomal organizer, which harbors HyWnt3 expressing cells is marked in red. (b) Cleavage of recombinant HyWnt3-His, monitored by Western blotting with anti-His antibody, reached half-maximal levels after $\sim 4 \mathrm{hrs}$ incubation in head lysate and after $\sim 6 \mathrm{hrs}$ in upper body lysate. No cleavage was observed during incubation in tentacle and lower body lysates, while incubation in the PBS control showed unspecific cleavage at $24 \mathrm{hrs}$. No unspecific proteolysis on $1 \mu \mathrm{g}$ BSA was detectable in $\mathrm{HL}$ over the time period of $24 \mathrm{hrs}$ as detected by SDS-PAGE and Coomassie staining. HyWnt3-His cleavage activity in head lysate was completely blocked by the addition of broad zinc metalloproteinase inhibitors EDTA and Phenanthroline or the matrix metalloproteinase inhibitor Batimastat. (c) No cleavage was observed for the recombinant Wnt antagonist HyDkk1/2/4-His in the respective body tissue lysates during a $24 \mathrm{hrs}$ incubation time. Mark that the double band appearance is an SDS-PAGE artifact. (d) Tissue lysates from different body parts of adult hydra polyps as indicated in the scheme were adjusted in total protein concentration by tubulin Western blotting. (e) Distribution of protein classes in Hydra head lysate secretome identified in HyWnt3(+) and HyWnt3(-) fractions as indicated. The full dataset is given in Additional file 4: Table S1a-b. (f) HyWnt3-His processing is inhibited by recombinant mouse Fetuin-B protein in a dose-dependent manner as indicated. 
bioRxiv preprint doi: https://doi.org/10.1101/2020.08.13.247569; this version posted August 13, 2020. The copyright holder for this preprint (which was not certified by peer review) is the author/funder. All rights reserved. No reuse allowed without permission.

Fig. 2

\section{a}

Asp switch

Astacin
NAS-35
ZHEE-1

Zn binding

Astacin AGVSG---ADQSAILSGMQELEEKTCIRFVPRT-------TESDYVEIFTSGSGCWSYVGRIS-----GA-QQVSLQANGCVYHGTIIHELMHAIGFYHEHTRM 156 NAS-35 DGSHT--QREQRIIELALEHWHNITCLNFQRNDQA-----NSGNRIVF-TDVDGCASNVGRPP----LGEEQLVSLAPE-CIRLGVIAHEVAHALGFWHEQSRP 243 HCE-1 SSEYS--GGEVATIEGAMPAFNGKTCIRFYRRT------NEYPFISVY-SKTGCYSELRRG-----GQ-OELSINRGGCMYSGITOHELNHALGFOHEQTRS 180 Meprin $\alpha$ ADNLG-LNAKGAILYAFEMFRLKSCVDFKPYE-------GESSYIIF-QPFDGCWSEVGDQH----VG--QNISIGQG-CAYKAIIEHEILHALGFYHEQSRT 176 Meprin $\beta$ EDSLE--MNAKGVILNAFERYRLKTCIDFKPWA-------GETNYISV-FKGSGCWSSVGNRR----VGK-OELSIGAN-CDRIATVQHEFLHALGFWHEQSRS 173 SPAN ESSSS--GQS-SLIRSAMDHWEQNTCLRFEPLTSSHSSRLGHTSYISF-FRGNGCWSHVGRSF----TNQ-QQISIGPQ-CGYFGTIVHEIGHAIGFHHEQSRP 211 HMP-1 DNNFD--NRRRQLVNQAITSYNQHTCVRFAPRT--------NQRDFVHMV-NEQGCWSIIGRQG-----NS-QKLKLGDW-CLDIGTAIHEMMHAAGFYHEQSRN 162 HAS-3 DRSLG-TQARKAIADAINVYHKVTCLKFIPRT-------SERAYINFF-FGDGCFSPVGYESSIWLNQK-NDVSIGSG-CESVGTVLHEVGHSIGLYHEQNRP 163 HAS-6 DNTID--AGTRQAINDAIADYHTYTCLRFVPRK-------SEPNYIRFT-KGAGCTSPVGMSG-----GV-NVVSLGEN-CGDKGTALHEIGHSIGLYHEQSRP 158 HAS-5 DNTLN--QTTRQAINDAIADYHTYTCLRFVPRI-------NQPNYIRFT-NGNGCSSPVGMSG-----GV-NVVTLGKG-CGDKGTALHEIGHSVGLHHEQSRP 159 HAS-4 ESSIG--ENGRQAIKEAIDDYHRFTCLRFTPWN--------GEKNYISFF-YGQGCNSPVGMWD-------K-NRISLGEG-CLTKGTALHEIGHSLGLEHEQCRP 159

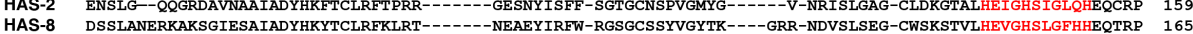
HAS-9 DSSLAKEKKARSGIESAIADYHKYTCLRFKORT--------NEVEYIREW-RGSGCSSPVGFTK----GRV-NDVSLSDG-CWGKATVLHEVGHSLGFHHEOTRP 165 HAS-1 SKMST---DAHOSILNAIADYQRRTCLKFVKRT--------NEPTYLSFF-VGNGCSSPVGYRQ----GRI-NTISLGRG-CLGHGTILHEIGHSIGLYHEQSRP 155 HAS-11 SRLNK---QSQPMVAKAIEEYHKHTCLKFVQRT-------NQREYLSFY-HGSGCSSPVGYQQ----YRV-NEVSLASG-CFKLGTVMHEIGHSMGFYHEQSRP 156 $\begin{array}{llll}\text { HAS-7 } & \text { RRMSS---SNHVYVLRAIDEYHKHTCLKFVKRT--------NEDAYLSFF-PGGGCSSLVGYMR----GRV-NEVSLAGG-CLQLGTVLHEIGHSIGLYHEQSRP } & 151 \\ \text { HAS-10 } & \text { SRMSS---SNHIHVLRAIDEYHKHTCLKFVRT-------NQDAYLSFY-PGGGCSSLVGYR----GRI-NDVSLAGG-CLRLGTVMHEIGHSIGLYHEQSRP } & 154\end{array}$ Met-turn $S_{1}$

Astacin DRDNYVTINYQNVDP----SMTSNFDIDT--YSRYVGEDYQYYS IMHYGKYSFSIQWGVLETIVPLQN-G-IDLTDPYDKAHMLQTDANQINNLYTNECSLRH- 251 NAS-35 DRDQYVTVRWENIDK----DSKGQFLKEDPDDVDNAGVPYDYGS IMHYRSKAFSKFDD-LYTISTYVTDYQKTIG---QRDQLSFNDIRLMNKIY---CSA--- 327 $\begin{array}{llll}\text { ZHE-1 DRDQYVINWNISP----GMAYNFLKQK---TNNQNTPYDYGSLMHYGKTAFAIQPG-LETITPIPDEN-VQIG---QRQGLSKIDILGINKLY--GC----- } & 263 \\ \text { HCE-1 } & \text { DRDYVRI } & \end{array}$

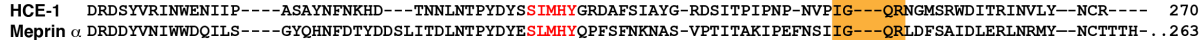

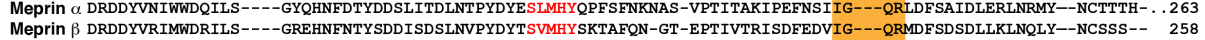
Meprin $\beta$ DRDDYRIMWDRILS----GREHNFNTYSDDISDSLNVPYDYTSVMHYSKTAFQN-GT-EPTIVTRISDFEDVIG---QRMDFSDSDLLKLNQLYY--NCSSS--
SPAN DRDEINVHFENVS---GREHNFAKYTWGSVTSSNVEYDVG IMHYGGGFSS-NG-RPTITTIDPRLNSRGG---QRTALSAADIELANRIY--ECDD--- 295 HMP-1 DRDDHVVIFWNNIQR----GEDYNFQKYR---TEYYGQTYDHNS IMHYRNDEFSAN-G-KNTIQAKNNP-GLKLG----NNQFSKSDIAAINQMY--KCSG--- 241 HAS-3 DRDRYVTIVNSNIMK----DM--DYNFQLEYDIDSLKTPYDLKS IMHYGSKAFAVNRN-LRTIVTKDPKLQYLIDHYDDVNNFFSWDIKQINLMY-PLCLGK-- 252 HAS-5 DRGRYVNINLNNIDP----KYGTAFAFDIANDIDSLGTEYDYRSAMHYSSEAFAKP-G-IQTITTVDPKNMKYIENFNQI IGFSVIDVQQIRKMY--NCPG--- 245 HAS-4 DRDRYVNIHYENIEE----DW--VFAFNISKNVDSLGTEYDLYSMMHYSSNSFTKVWN-LKTITTVDRNMKYIETYNQIIGFSVVDVQQIRLMY--SCPA--- 246 HAS-2 DRDQHVIIHTKNIDG----PW--AYAFAISNEVDSLGTPYDLSSMMHYSSTAFAKP-N-TKTITTKDPSKQKLIDNYNRIFGFSSTDIVQINKMY--SCGKKLI 246 HAS-8 DRDSFVTIVKSNISP----GTYFNFEKENARDINSYGSPYDYLSMMHYSWNAFAVNTN-SPSIITLNKEYQYHIG---QDEGFSRSDVIQLNKMY--RCSGS-- 249 HAS-9 DRDKYVIIVSNIHS----DTLFNEEESEKKINSHGTTYDYTS MMHYANAFAINTE-SPSIITLNKEYQYHIG---QDEGFSKTDVIQLNKMY--KCSGN-- 249 HAS-1 DRDNYVTIVWNNIQK----EMQFNFNK--ETNIDSLQTSYDYNS IMHYGATAFGNG---KSTIITKDPSKQQVIG---NRSGFSTIDVQQINAMY--NCKMG-- 238

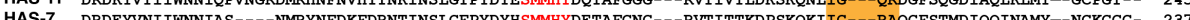
HAS-10 DRDDHVTIIWNNIOS-----NMRFNFDKFDRNKINSLGFPYDYESMMHYDOTAFGGG---OVTIRTKDPSKOKLIG---NROGFSEIDKOOINAMY--NCNRG-- 239

b

HAS-1, HAS-2, HAS-3, HAS-4 Pro Protease

HMP-1, HAS-5, HAS-6

Protease ShKT

HAS-7, HAS-8, HAS-9, HAS-10 Pro Protease

HAS-11

Protease ShKT ShKT ShKT ShKT ShKT ShKT

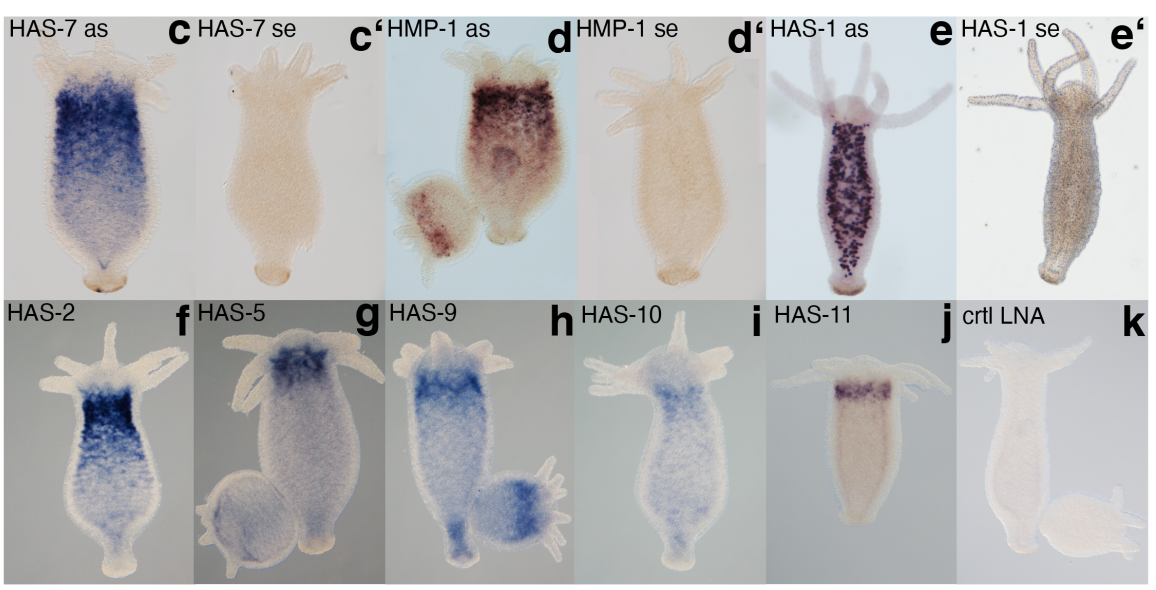


Fig. 2. Sequence features and expression patterns of HyWnt3(+) astacin genes.

(a) Multiple sequence alignment of pro-domain and catalytic domain sequences of astacins identified in this study. For comparison, astacin sequences from diverse species outside the cnidarian phylum were included. Gene ID numbers are as follows: Astacin A. astacus (P07584), NAS-35 C. elegans (P98060), ZHE-1 Danio rerio (Q1LW01), HCE-1 O. latipes (P31580), Meprin $\alpha H$. sapiens (Q16819), Meprin $\beta$ H. sapiens (Q16820), SPAN S. purpuratus (P98068), HMP1 (NP_001296695.1), HAS-3 (XP_002166229.3), HAS-6 (XP_002157397.2), HAS-5 (XP_002164800.1), HAS-4 (XP_002162738.1), HAS-2 (XP_002162822.1), HAS-8 (XP_002153855.1), HAS-9 (XP_002161766.1), HAS-1 (XP_012565441.1), HAS-11(XP_012561076.1), HAS-7 (XP_012560086.1), HAS-10 (XP_002159980.2). In red: the aspartate switch residue in the pro-peptide, the zinc binding motif and the Met-turn. Orange background: residues forming the $\mathrm{S} 1$ ' sub-site. - denotes the activation site. (b) Domain structures of astacins detected in HyWnt3(+) head lysate fractions. S, signal peptide; Pro, prodomain; ShKT, Stichodactyla toxin domain. (c-k) WISH experiments using antisense and sense oligonucleotide probes (c-e') or LNAs (f-k) show a collar-like expression pattern marking a transition zone between head and body column for the majority of astacin genes. Representative of 10 hydras examined. 
Fig.3
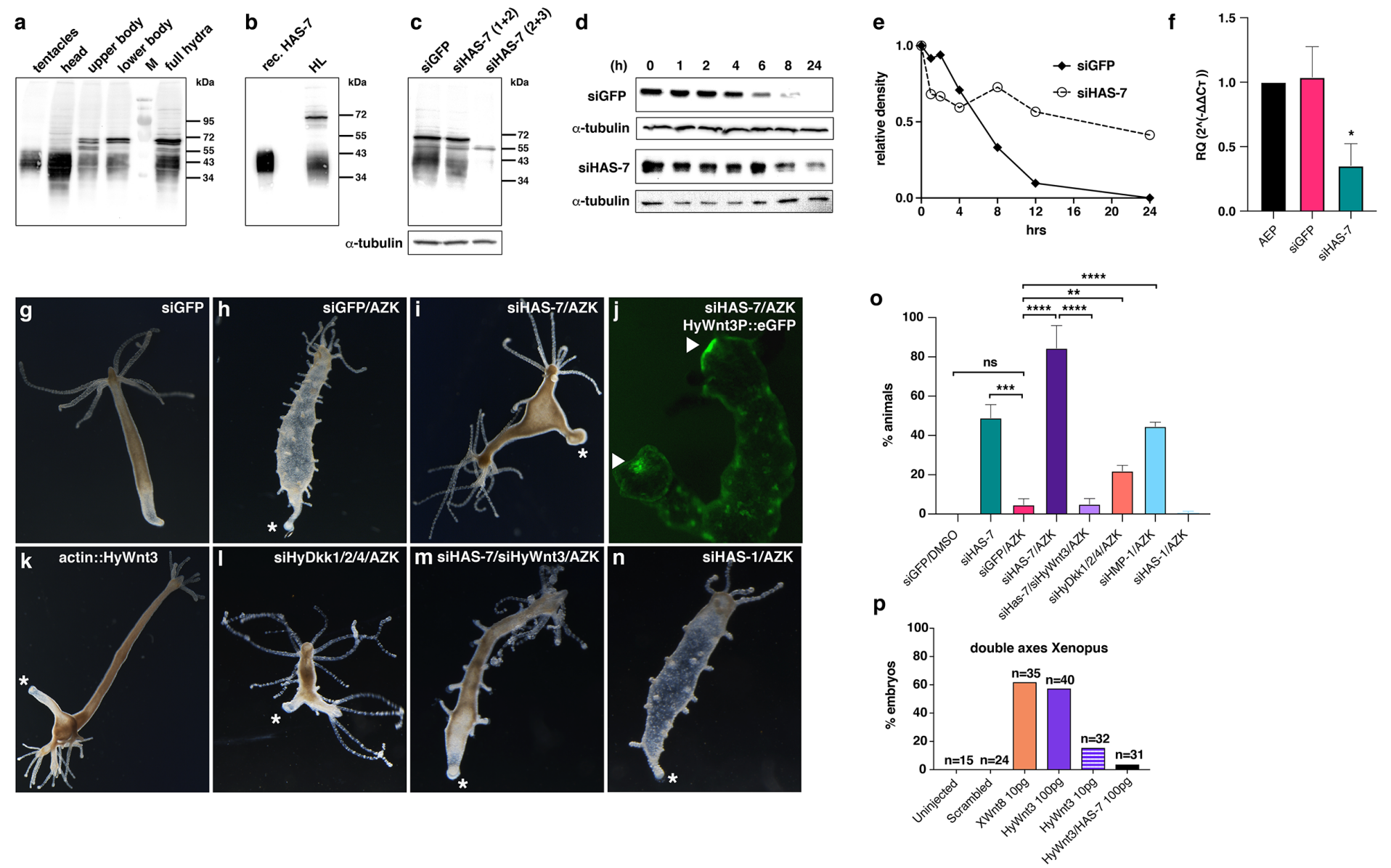
Fig. 3. Functional analysis of HAS-7 knockdown. (a) Detection of HAS-7 protein in body lysate samples using a polyclonal HAS-7 antibody. $M=$ protein marker. (b) Recombinant HAS-7 protein expressed in High Five cells compared to native protein in head lysate as detected by HAS-7-specific antibody. Note that that the slightly higher apparent molecular mass is due to the introduced histidine tag of recombinant HAS-7. (c) Knockdown effect of different siRNA combinations on HAS-7 protein levels was assayed by anti-HAS-7 Western blot analysis of complete hydras treated as indicated. Tubulin was used as loading control of the respective hydra lysates. The distinct band at $70 \mathrm{kDa}$ in a-c likely represents a dimer of processed HAS-7. (d) HyWnt3-His proteolysis is impaired in head lysate of animals electroporated with siHAS-7 (2+3) as compared to siGFP control animals. Head lysates were generated 6 days after electroporation. Tubulin was used as loading control of the respective head lysates applied for each time point. (e) Relative intensities of the Western blot bands in d. (f) Quantitative real-time PCR analysis of HAS-7 expression in head tissues confirms the decreased expression in siHAS-7 treated animals compared to siGFP treated and untreated (steady-state AEP animals) controls. Results represent mean +/- S.D. from 3 independent experiments, analyzed by $t$ tests. ${ }^{*} p<0.05$. (g) siGFP control electroporation without AZK treatment shows normal morphology. (h) siGFP control animal showing ectopic tentacle formation after AZK treatment. (i) Double axis phenotype in hydras treated with AZK after HAS-7 (2+3)/GFP siRNA electroporation. Asterisk denotes the peduncle region. (j) Both heads in HyWnt3P::HyWnt3 transgenic animal treated as in $\mathrm{h}$ exhibit hypostomal HyWnt3 expression (arrow). Smaller spots along the body column represent ectopic organizers that usually give rise to ectopic tentacles as in h. (k) Double axis phenotype in actin::HyWnt3 transgenic hydra. (I) Double axis phenotype in hydras after HyDkk1/2/4/GFP siRNA electroporation and AZK treatment. $(m)$ Rescue of double axis phenotype in animals treated with AZK after electroporation with a combination of HAS-7 $(2+3)$ and HyWnt3/GFP siRNAs. (n) No double axes were observed in hydras treated with AZK after electroporation with HAS1/GFP siRNA. (o) Ratios of double axis phenotypes in hydras after electroporation with siGFP or combinations of siGFP with siRNAs as indicated. In animals without subsequent AZK treatment double axes were counted 6 days after electroporation. In animals treated additionally with AZK, incubation was started 6 days after electroporation and the numbers of double axes in each group were counted 5 days 
after AZK removal. Animals (n) in each group were: siGFP/DMSO = 192, siGFP/AZK $=230$, siHas-7/siGFP $=186$, siHAS-7 /siGFP/AZK $=248$, siHAS-7 /siHyWnt3/siGFP/AZK = 203, siHyDkk1/2/4/siGFP/AZK = 290, siHMP-1/siGFP/AZK = 204, siHAS-1/siGFP/AZK = 150. Results from at least three independent experiments are shown. Each column represents the total percentage of one group, bars indicate the mean \pm S.E.M. ${ }^{\star \star * \star} P$ value $<0.0001,{ }^{* \star *} \mathrm{P}$ value $<0.0005,{ }^{\star \star} \mathrm{P}$ value $<0.001$. ns $=$ not significant. The data were analyzed using an unpaired parametric T-test with Welch's correction followed by pairwise multiple comparisons of each group with the other groups. ( $p$ ) Coinjection of HAS-7 mRNA inhibited HyWnt3 mRNA induced ectopic axis in Xenopus laevis axial duplication assay. Xenopus Wnt8 (XWnt8) mRNA injection served as positive control. Injected mRNA doses were as indicated. $\mathrm{n}$ indicates the number of embryos analyzed for each experimental condition. 
Fig. 4

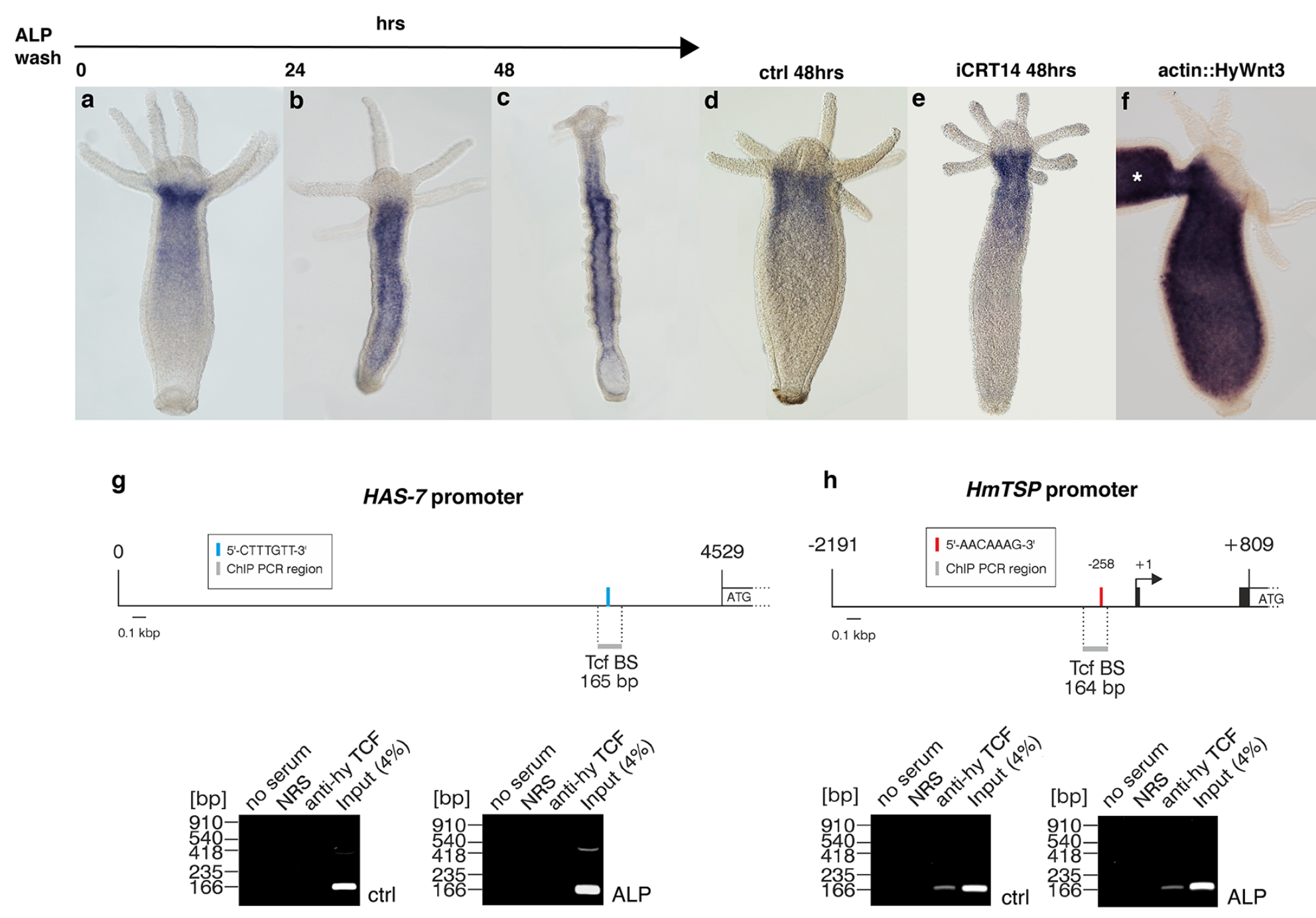


Fig. 4. $\beta$-Catenin dependent expression of HAS-7. (a-c) ISH analysis of HAS-7 expression after ALP treatment shows a global upregulation after $24 \mathrm{hrs}$ and a shift towards the developing ectopic organizers along the body column after $48 \mathrm{hrs}$ as compared to DMSO-treated controls (d). At Oh after ALP wash (a) no change of the HAS-7 expression pattern compared to untreated controls (compare d and Fig. 2a) was evident. (e) Inhibition of beta-Catenin activity by iCRT14 treatment does not reduce normal HAS-7 expression levels. (f) HAS-7 expression is globally upregulated in the gastric region of transgenic actin::HyWnt3 animals. The asterisk marks a secondary axis. Representatives of 10 hydras examined.

(g-h) No detectable binding of Hydra TCF to the HAS-7 promoter. (g) ChIP analysis of the Hydra magnipapillata HAS-7 promoter. Upper site: Topography of the HAS-7 5'untranslated region (nt 1 to 4529). The ATG indicates the translation start site. The position of a canonical TCF binding motif (5'-CTTTGTT-3') is indicated by a blue bar. The localization of the 165-bp DNA segment flanked by the specific ChIP primer pair is visualized with a grey bar. Lower site: ChIP analysis of the Hydra HAS-7 promoter region using chromatin from untreated whole hydra animals (ctrl), and from animals treated with ALP. A polyclonal antibody directed against Hydra TCF was used for precipitation, followed by PCR amplification of the indicated fragment from the HAS-7 regulatory region. Reactions with normal rabbit serum (NRS) or total chromatin (Input) were used as controls. PCR products were resolved by agarose gel electrophoresis, and visualized by ethidium bromide staining. (h) ChIP analysis of the HmTSP promoter performed under the same conditions as in ( $\mathrm{g}$ ) and used as a positive control. Upper site: Topography of the 3,000-bp HmTSP promoter (nt -2191 to +809 ). Black boxes depict the first two exons of the HmTSP gene. The arrow indicates the transcription start site of the HmTSP mRNA, and ATG the translation start site. The position of the tested canonical TCF binding motif (5'-AACAAAG-3') is indicated by a red bar. The localization and size of a 164-bp DNA segment flanked by specific the specific ChIP primer pair is visualized with a grey bar. Lower site: ChIP analysis as described under (g). 
Fig. 5

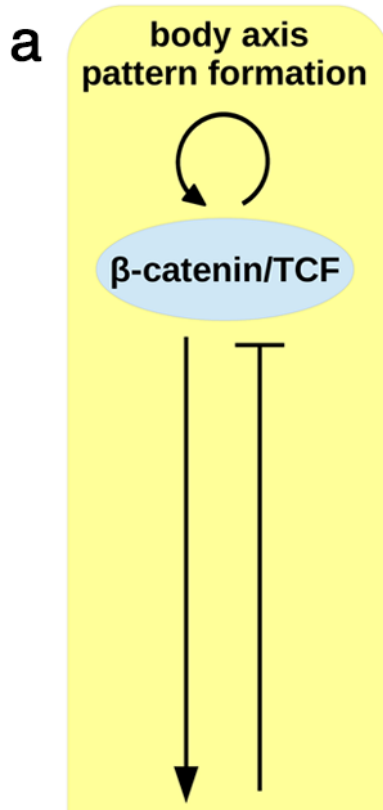

HyDkk12/4
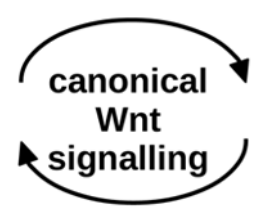

body axis gradient long-term storage

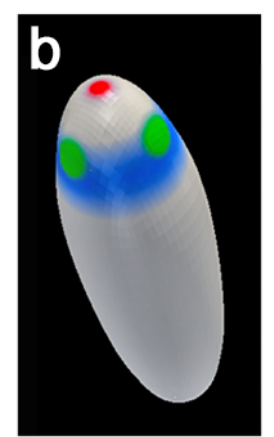

undisturbed system

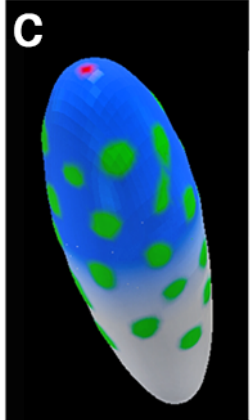

AZK

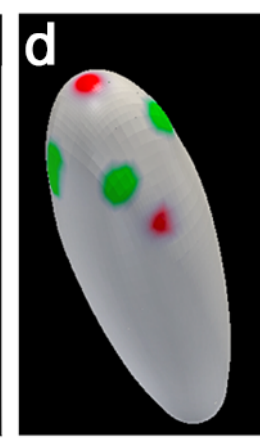

AZK + siHAS-7

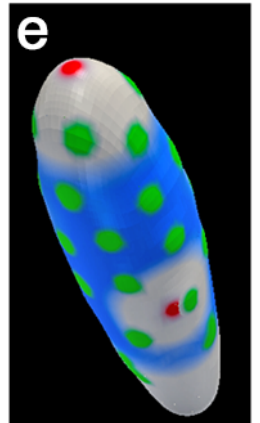

AZK + siDkk1/2/4

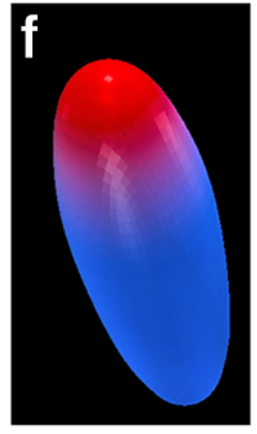

high HyWnt3

Fig. 5. Mathematical model of HAS-7 function. (a) schematic representation of the model. Yellow blocks depict self-containing pattern formation systems consisting of an activator and an inhibitor. (b)-(f) finite element simulations of different experimental scenarios. Blue color corresponds to high levels of HAS-7 expression, red color to HyWnt3 expression, and green color indicates tentacle formation. The scale of these expression intensities is similar across all simulation plots. 
Fig. 6

a

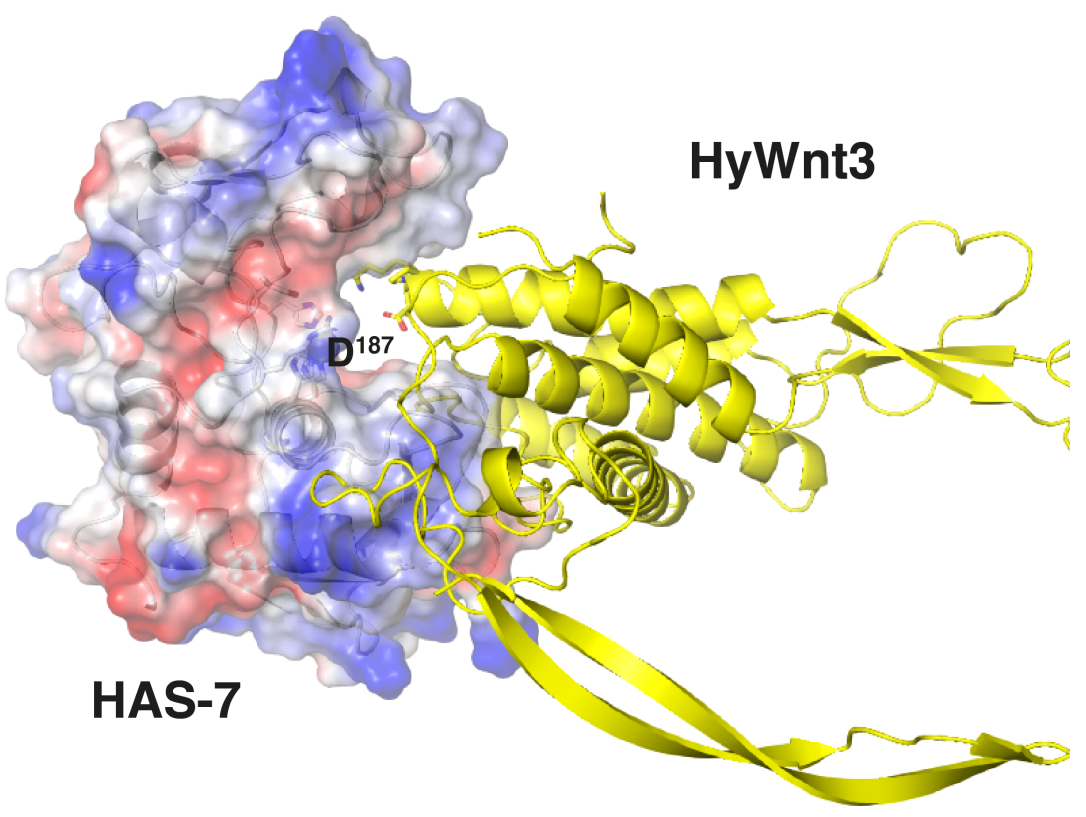

b

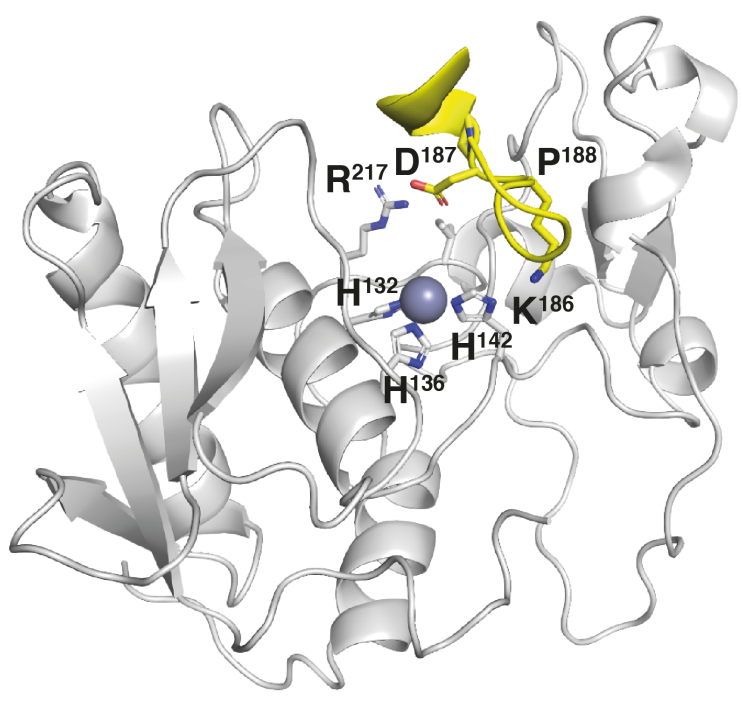

C

Fig. 6. Structural model of the putative HyWnt3:HAS-7 complex. (a) Overview of HAS-7 (surface potential) complexed with HyWnt3 (yellow cartoon presentation). The active site $\mathrm{Zn}$-ion of the astacin is shown as pink sphere. (b) Detailed view inside the active site pocket, shown in standard presentation with the $\mathrm{Zn}$-ion located at the bottom of the cleft. (c) Alignment of amino acid sequences in different Hydra Wnt proteins encompassing the putative DP cleavage motif in HyWnt3. 